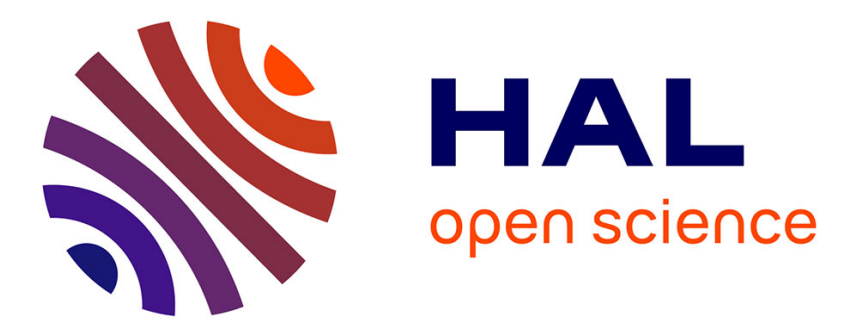

\title{
Combining planar and central chirality in ferrocene thiophosphine-sulfoxides
}

\author{
Raluca Malacea, Jean-Claude Daran, Rinaldo Poli, Eric Manoury
}

\section{To cite this version:}

Raluca Malacea, Jean-Claude Daran, Rinaldo Poli, Eric Manoury. Combining planar and central chirality in ferrocene thiophosphine-sulfoxides. Tetrahedron: Asymmetry, 2013, 24 (9-10), pp.612620. 10.1016/j.tetasy.2013.04.002 . hal-02908226

\section{HAL Id: hal-02908226 \\ https://hal.science/hal-02908226}

Submitted on 2 Mar 2021

HAL is a multi-disciplinary open access archive for the deposit and dissemination of scientific research documents, whether they are published or not. The documents may come from teaching and research institutions in France or abroad, or from public or private research centers.
L'archive ouverte pluridisciplinaire HAL, est destinée au dépôt et à la diffusion de documents scientifiques de niveau recherche, publiés ou non, émanant des établissements d'enseignement et de recherche français ou étrangers, des laboratoires publics ou privés. 


\section{Combining planar and central chirality in ferrocene thiophosphine-sulfoxides}

RalucaMalacea, ${ }^{\mathrm{a}, \mathrm{b}}$ Jean-Claude Daran, ${ }^{\mathrm{a}, \mathrm{b}}$ Rinaldo Poli, ${ }^{\mathrm{a}, \mathrm{b}, \mathrm{c}}$ Eric Manoury ${ }^{\mathrm{a}, \mathrm{b}} *$

a CNRS, LCC (Laboratoire de Chimie de Coordination), 205 route de Narbonne, BP 44099, F-31077

Toulouse Cedex 4, France; Fax: (+) 33-561553003; E-mail: manoury@lcc-toulouse.fr

${ }^{b}$ Université de Toulouse, UPS, INPT, F-31077 Toulouse Cedex 4, France

Institut Universitaire de France, 103, bd Saint-Michel, 75005 Paris, France 


\begin{abstract}
New chiral ferrocenylthiophosphine-sulfoxide and phosphinesulfoxidederivativespossessing planar chirality for theferrocenemoietyand central chirality at the sulfur atomhave been synthesized. These ligands could be obtained as pure diastereoisomersinboth racemic andenantiomerically pure forms. Complete characterization by XRD analysis has allowed the absolute configuration assignmentineachcase. Preliminarycoordination studies of the phosphine-sulfoxideligandson platinum are also reported. They show chelating complexationbythe phosphorous and sulfur atoms.
\end{abstract}

\title{
Image for the graphical abstracts:
}

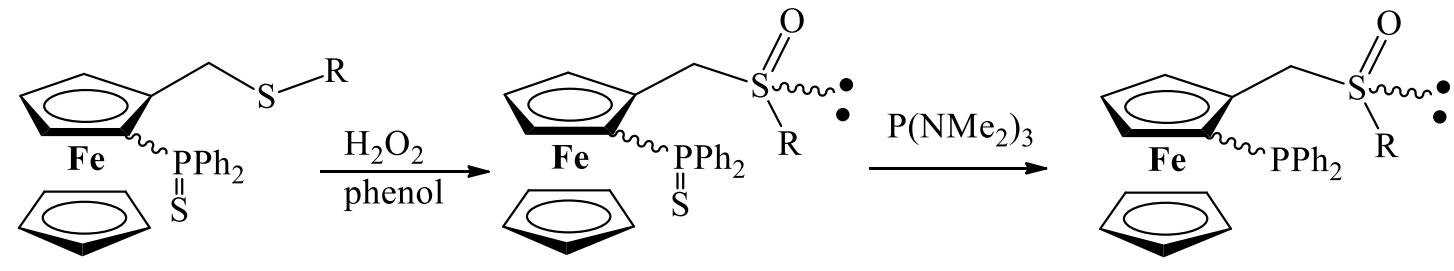

$\mathrm{R}=\mathbf{P h}, \mathbf{E t}, \boldsymbol{t B u}$ 


\section{Introduction}

Various chiral bidentate ligands were developed over the past decades, possessing the chiral information on the carbon backbone or on the donor atom. Ligands with variouscombinations of donor atoms (in particular, $\mathrm{P}-\mathrm{P}, \mathrm{P}-\mathrm{N}$, or $\mathrm{N}-\mathrm{N}$ ligands) have been extensively studied and a fewP-chirogenic compounds such as Quinox $\mathrm{P}^{1}$ or dipamp ${ }^{2}$ are very effective in a variety of asymmetric catalytic processes. Recently, very efficient systems for asymmetric catalysis using P-S ligands with various backbones, havebeen reported. ${ }^{3,4}$ Furthermore, several reports have described the preparation and the coordination chemistry of chiral phosphine sulfoxide ligands possessing the chirality on the sulfur atom, for example $\alpha$ phosphinosulfoxides $\left(\mathrm{PCSO}^{5}\right.$ andPNSO$^{6}$ ) or $\beta$-phosphinosulfoxides ${ }^{7}$ (Figure 1). Riera and Verdaguer $^{8}$ described ligands with an extra chiral centerin the central carbon atom between $\mathrm{P}$ and $\mathrm{SO}$ and also camphor derivatives combininga chiral carbon backbone with sulfur chirality. ${ }^{9}$ Kagan's methodology based on diastereoselectiveortho-lithiation of ferrocenesulfoxide derivatives was used in order to synthesize phosphine-sulfoxide ligands with additional planar chirality. ${ }^{10} \mathrm{We}$ have recently developed syntheses of new chiral ferrocenyl $\gamma$-P,S ligands in both racemic and enantiomerically pure form $\left(R_{\mathrm{Fc}}\right.$ or $S_{\mathrm{Fc}}$ configuration), and reported on their coordination chemistry and applications to asymmetric catalysis, namely in asymmetric allylic substitution, alkene methoxycarbonylationand ketone hydrogenation. ${ }^{11}$ Here we present the synthesis of new $\gamma$ ferrocenyl phosphine sulfoxide ligands (Figure 1)and preliminary studies on their coordination chemistry with platinum.

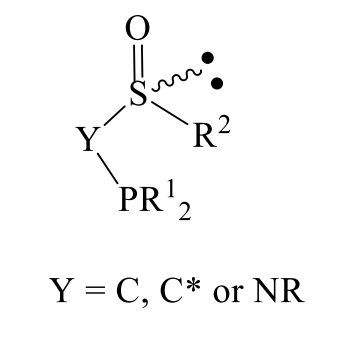

$\alpha$-phosphine sulfoxides
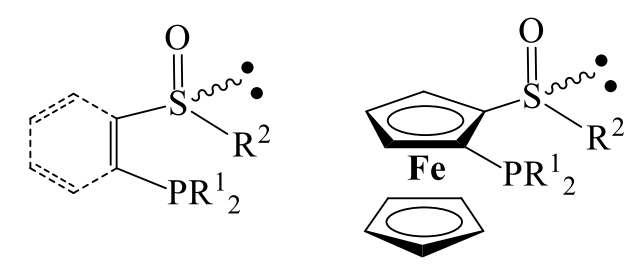

$\beta$-phosphine sulfoxides

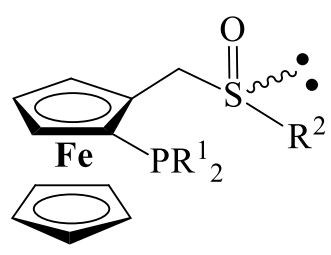

$\gamma$-phosphine sulfoxides

Figure $1 \alpha, \beta$ and $\gamma$ phosphine sulfoxides

\section{Results and Discussion}

Starting from the chiral phosphine-thioetherligands already synthesized in our group, we have developed a new class of ferrocenyl phosphine-sulfoxides ligands based on the selective oxidation of the thioether functionality to sulfoxide. Thus, a central chirality on the sulfur atom $\left(R_{\mathrm{S}}\right.$ or $\left.S_{\mathrm{S}}\right)$ is added to the planar chirality of ferrocene $\left(R_{\mathrm{Fc}}\right.$ or $\left.S_{\mathrm{Fc}}\right)$. The two diastereoisomersthatcan a prioribe formed must therefore be subsequently separated.

Afirst potential issue in the synthetic pathway to the thiophosphine-sulfoxides 2 fromthiophosphine-thioethers1(see scheme 1) is the possible over-oxidation to the corresponding sulfonesas well as oxidation of the iron atom to ferroceniumor conversion of 
thethiophosphine to phosphine oxide, as already observed in the oxidation of related (2diphenylthiophosphinoferrocenyl)methanol to the corresponding aldehyde. ${ }^{12}$ Therefore, the mild method described by Liang $\mathrm{Xu}^{13}$ using an aqueous solution of $\mathrm{H}_{2} \mathrm{O}_{2}$ in the presence of phenol was selected.Under these conditions, the racemic compounds 1a-c $(\mathrm{R}=\mathrm{Ph}, \mathrm{Et}, t \mathrm{Bu})$ were transformed into the corresponding thiophosphine-sulfoxides $\mathbf{2 a - c}$ with total conversion and chemoselectivity (no sulfoneor other by-products were observed). For each thioether, two diastereoisomers (each one as a pair of enantiomers)were obtained in a 1:1 ratio. These reactions are fast (2-3 minutes) andstrongly exothermic. For safety reasons, we therefore limited the thioetheramount (typically $250 \mathrm{mg}$ ) transformedineach run. Eachdiastereomercould be isolated in good yields after separation by column chromatography (Scheme 1).

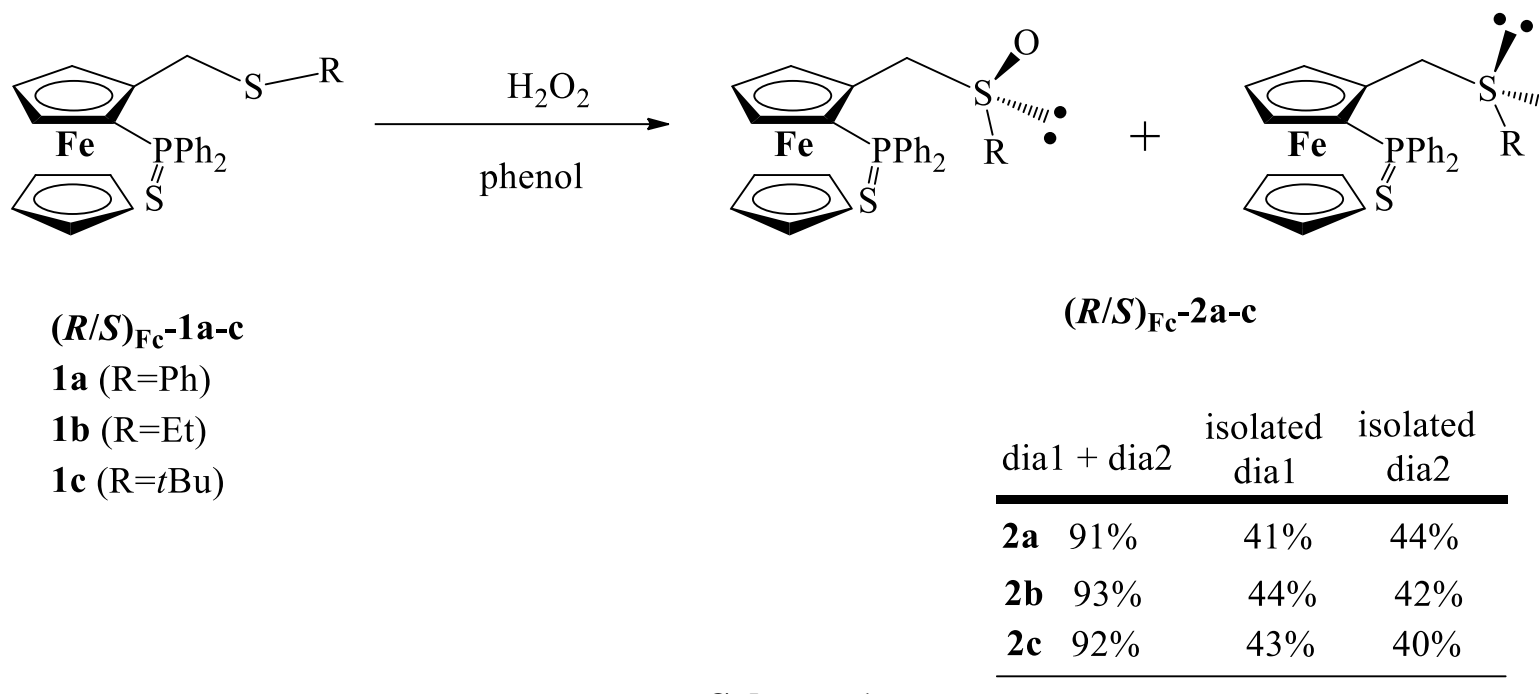

Scheme 1

After diastereomerseparation, the sulfur atomconfigurations were established by single crystal X-ray diffraction analyses. Single crystals of 2a-Dia1, 2a-Dia2, 2b-Dia1, 2c-Dia1 and $2 \mathrm{c}$-Dia2 2 were obtained by slow diffusion of pentane vapors into a dichloromethane solution. Theseproductswere obtained from the racemic thiophosphine-thioethers1a-c $\left(R / S_{\mathrm{Fc}}\right)$ and crystallize as racemates, except for 2c-Dia2 which affords a twin by inversion, therefore also containing both enantiomers (70:30) in the crystal.
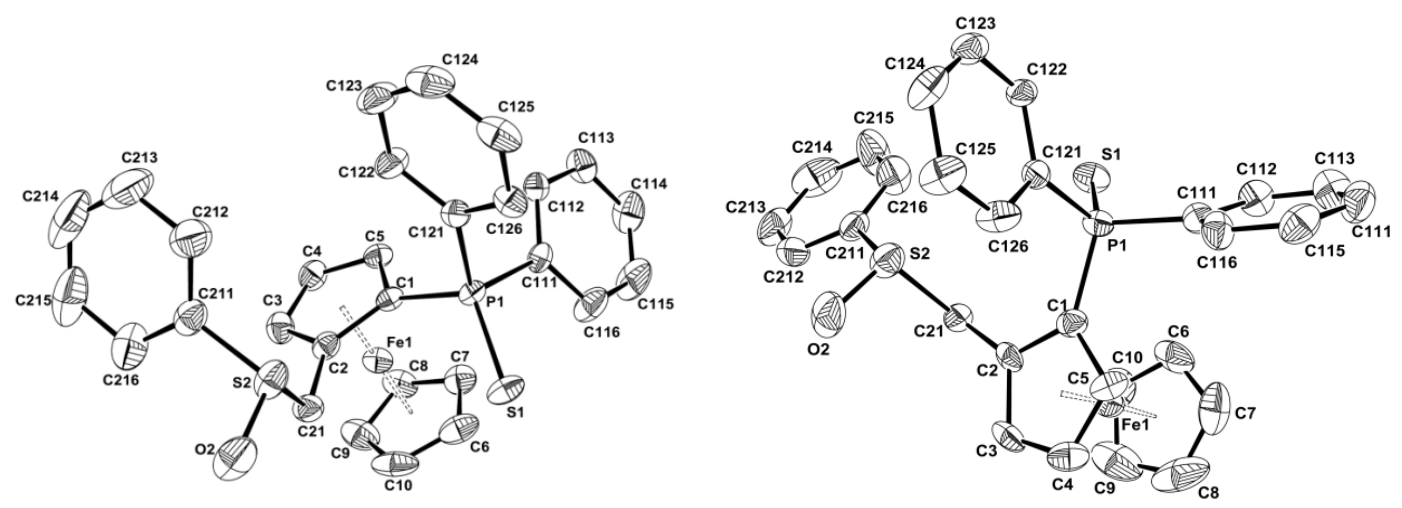


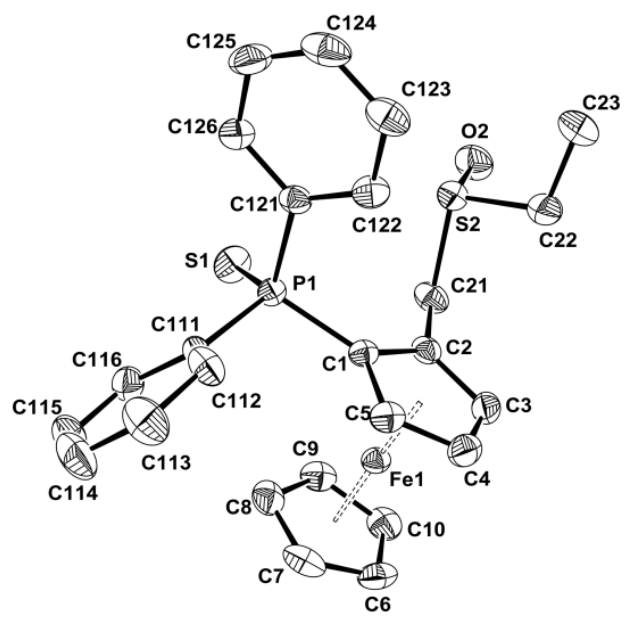

2b-Dia1 $\left(R_{\mathrm{Fc}}, R_{\mathrm{S}}\right) /(*)$

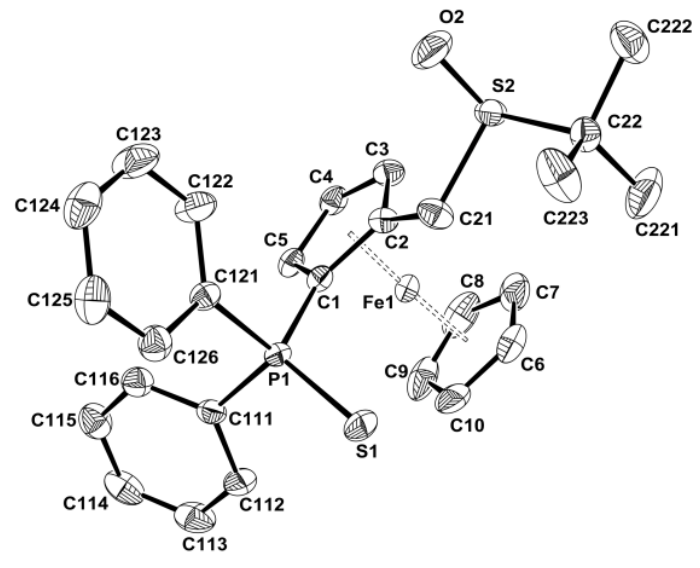

2c-Dia1 $\left(S_{\mathrm{Fc}}, R_{\mathrm{S}}\right) /(*)$

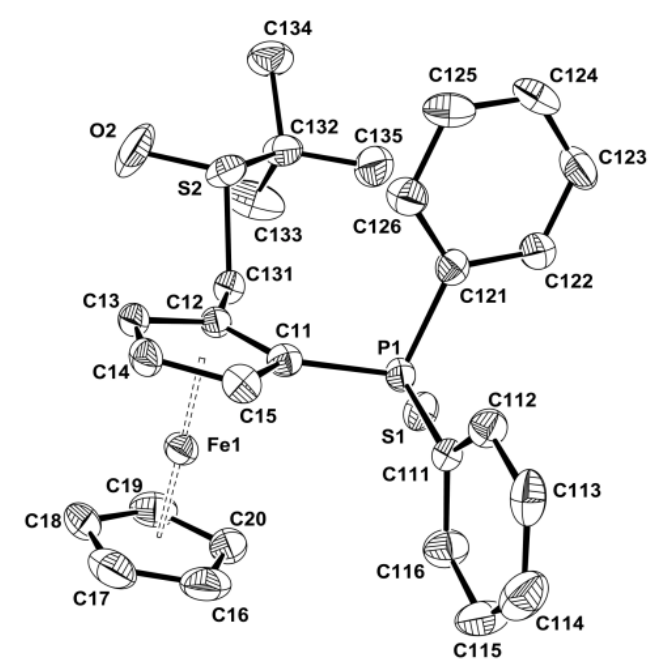

2c-Dia2 $\left(S_{\mathrm{Fc}}, S_{\mathrm{S}}\right) /(*)$

Figure 2.ORTEP views of2a-Dia1, 2a-Dia2, 2b-Dia1, 2c-Dia1 and 2c-Dia2 (* the other enantiomer is present in the crystal structure but not represented in the picture)with the atom labeling scheme. Ellipsoids are drawn at the 50\% probability level. $\mathrm{H}$ atoms have been omitted for the sake of clarity.

To the best of our knowledge, these compounds are the first structurally characterized ferrocenylthiophosphine-sulfoxides.Selected geometrical parameters are reported in Table 1. The five compounds have similar structures with the protecting $\mathrm{S}(1)$ endo with respect to the $\mathrm{Cp}$ ring whereas the sulfido $\mathrm{S}(2)$ is exo (Table 1). The distances and angles within the sulfoxidemoietyare identical within experimental error. The two $\mathrm{Cp}$ rings are either nearly 
eclipsed for 2a-Dia2, 2c-Dia1and 2c-Dia2B and in between eclipsed-staggered for 2aDia1,2b-Dia1 and 2c-Dia2A ( $\tau$ values in Table 1).In compound 2c-Dia2, there are two molecules within the asymmetric unit. These molecules have similar configuration as indicated by the molecular fitting shown in Figure 3. The only marked difference is the twist angles observed between the two Cp rings, $12.7(3)^{\circ}$ for molecule A and $4.5(3)^{\circ}$ for B.

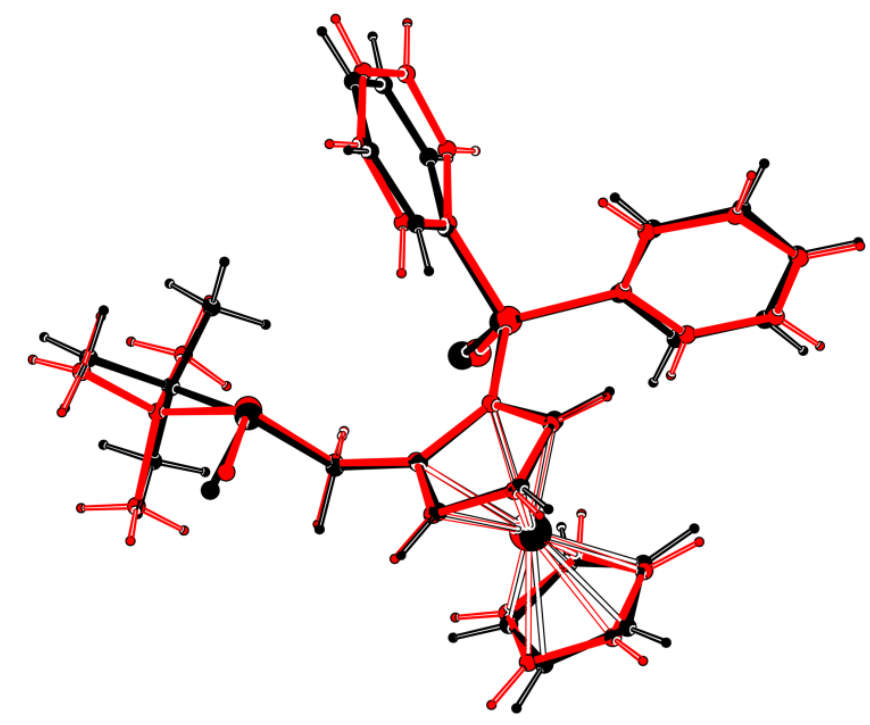

Figure 3.Molecular fitting of the two molecules 2c-Dia2A and 2c-Dia2B.

Table 1.Selected geometrical parameters for the structures of compounds 2,3 and 4 .

\begin{tabular}{|c|c|c|c|c|c|c|c|c|}
\hline & 2a-Dia1 & 2a-Dia2 & 2b-Dia1 & 2c-Dia1 & 2c-Dia2A & 2c-Dia2B & 3a-Dia2 ${ }^{a}$ & $4 a^{b}$ \\
\hline \multicolumn{9}{|l|}{ Distances $(\AA)$} \\
\hline $\mathrm{Fe}(1)-\mathrm{Cp}(1)$ & $1.6440(11)$ & $1.642(4)$ & $1.635(1)$ & $1.640(1)$ & $1.642(2)$ & $1.644(2)$ & $1.639(1)$ & $1.646(2)$ \\
\hline $\mathrm{Fe}(1)-\mathrm{Cp}(2)$ & $1.6582(14)$ & $1.654(6)$ & $1.652(2)$ & $1.655(1)$ & $1.655(2)$ & $1.659(2)$ & $1.653(1)$ & $1.661(3)$ \\
\hline $\mathrm{C}(1)-\mathrm{P}(1)$ & $1.788(2)$ & $1.788(8)$ & $1.802(3)$ & $1.792(2)$ & $1.788(4)$ & $1.798(4)$ & $1.816(2)$ & $1.796(5)$ \\
\hline $\mathrm{P}(1)-\mathrm{S}(1)$ & $1.9611(8)$ & $1.958(3)$ & $1.957(1)$ & $1.9584(8)$ & $1.958(2)$ & $1.956(2)$ & & \\
\hline $\mathrm{C}(2)-\mathrm{C}(21)$ & $1.491(3)$ & $1.502(11)$ & $1.502(4)$ & $1.500(3)$ & $1.496(5)$ & $1.495(5)$ & $1.487(3)$ & $1.481(8)$ \\
\hline$C(21)-S(2)$ & $1.817(2)$ & $1.828(8)$ & $1.825(3)$ & $1.811(3)$ & $1.820(4)$ & $1.817(4)$ & $1.823(2)$ & $1.810(5)$ \\
\hline $\mathrm{S}(2)-\mathrm{O}(2)$ & $1.499(2)$ & $1.485(6)$ & $1.508(2)$ & $1.494(2)$ & $1.495(4)$ & $1.480(4)$ & $1.470(2)$ & $1.459(3)$ \\
\hline $\mathrm{S}(2)-\mathrm{C}(22)$ & $1.782(3)$ & $1.787(9)$ & $1.803(3)$ & $1.841(3)$ & $1.826(5)$ & $1.840(4)$ & $1.790(2)$ & $1.782(6)$ \\
\hline \multicolumn{9}{|l|}{ Angles $\left(^{\circ}\right)$} \\
\hline $\begin{array}{l}\mathrm{Cp}(1)-\mathrm{Fe}(1)- \\
\mathrm{Cp}(2)\end{array}$ & $178.53(7)$ & $177.7(2)$ & $177.29(8)$ & $177.42(7)$ & $178.5(1)$ & $178.2(1)$ & $177.90(5)$ & 176.21(13) \\
\hline $\begin{array}{l}\mathrm{C}(2)-\mathrm{C}(21)- \\
\mathrm{S}(2)\end{array}$ & $115.90(17)$ & $111.0(5)$ & $113.1(2)$ & $110.5(2)$ & 108.1(3) & $107.5(3)$ & $110.3(1)$ & $116.2(3)$ \\
\hline $\begin{array}{l}\mathrm{C}(21)-\mathrm{S}(2)- \\
\mathrm{O}(2)\end{array}$ & $103.28(13)$ & $105.5(4)$ & $105.5(1)$ & $105.0(1)$ & $103.8(2)$ & $105.8(2)$ & $105.6(1)$ & $108.1(2)$ \\
\hline $\begin{array}{l}\mathrm{C}(21)-\mathrm{S}(2)- \\
\mathrm{C}(22)\end{array}$ & $100.84(12)$ & $94.4(4)$ & $97.8(2)$ & $101.0(1)$ & $100.4(2)$ & $100.6(2)$ & $95.65(9)$ & $97.5(2)$ \\
\hline $\begin{array}{l}\mathrm{O}(2)-\mathrm{S}(2)- \\
\mathrm{C}(22)\end{array}$ & $107.50(13)$ & $106.8(4)$ & $106.9(2)$ & $108.0(1)$ & $108.0(2)$ & $108.0(2)$ & $107.5(1)$ & $109.1(2)$ \\
\hline \multicolumn{9}{|c|}{ Distances from plane $(\AA)$} \\
\hline $\mathrm{S}(1)---\mathrm{Cp}(1)$ & $-0.859(1)$ & $-1.06(2)$ & $-0.921(6)$ & $-1.063(5)$ & $-0.992(8)$ & $-1.154(9)$ & & \\
\hline $\mathrm{S}(2)---\mathrm{Cp}(1)$ & $1.500(1)$ & $1.63(1)$ & $1.647(5)$ & $0.457(5)$ & $1.751(7)$ & $1.757(7)$ & $1.619(4)$ & $0.970(9)$ \\
\hline $\mathrm{O}(2)---\mathrm{Cp}(1)$ & $1.228(2)$ & $2.49(1)$ & $1.619(8)$ & $1.945(5)$ & $1.654(9)$ & $1.895(8)$ & $2.236(4)$ & $2.186(9)$ \\
\hline \multicolumn{9}{|c|}{ Twist angle for therCp rings $\left({ }^{\circ}\right)$} \\
\hline$\tau^{\circ}$ & $12.5(2)$ & $4.0(9)$ & $13.3(2)$ & $1.6(3)$ & $12.7(3)$ & $4.5(3)$ & $4.7(2)$ & $14.5(3)$ \\
\hline
\end{tabular}


As stated above, no diastereoselectivity was observed under theseoxidation conditions. Other achiral oxidants such as meta-chloroperbenzoic $\operatorname{acid}^{14}, t \mathrm{BuOOH} / \mathrm{MoO}_{2}(\mathrm{acac})_{2}{ }^{15}$ and also chiral ones such as $t \mathrm{BuOOH} /$ diethyltartrate/Ti $(\mathrm{O} i \mathrm{Pr})_{4}{ }^{16}$ and $t \mathrm{BuOOH} /$ diethyltartrate $/ \mathrm{MoO}_{2}(\mathrm{acac})_{2}$ were then employed. Unfortunately, all of them gave lower conversionsand no significant diastereoselectivity improvement relative to Xu's method. Therefore, our investigations werecontinued using the $\mathrm{H}_{2} \mathrm{O}_{2} /$ phenol system. The next step was the oxidation ofenantiomerically pure thiophosphine-thioethers $\left(R_{\mathrm{Fc}}\right)$ 1a-c 11a, 12which gave the corresponding thiophosphine-sulfoxides2a-c as a mixture of two diastereomers in enantiomerically pure form (Scheme 2). The results obtained working with optically pure thioethersare quite similar to those obtained using the compounds in racemic form. Thus, we have access to the desiredferrocenylthiophosphine-sulfoxidesin diastereoisomerically and enantiomerically pure form.

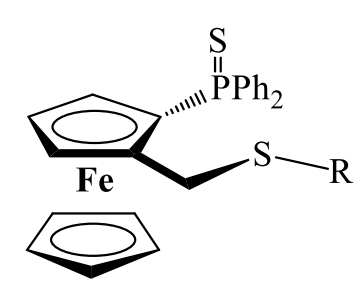

$\left(R_{\text {Fc }}\right)$ 1a-c

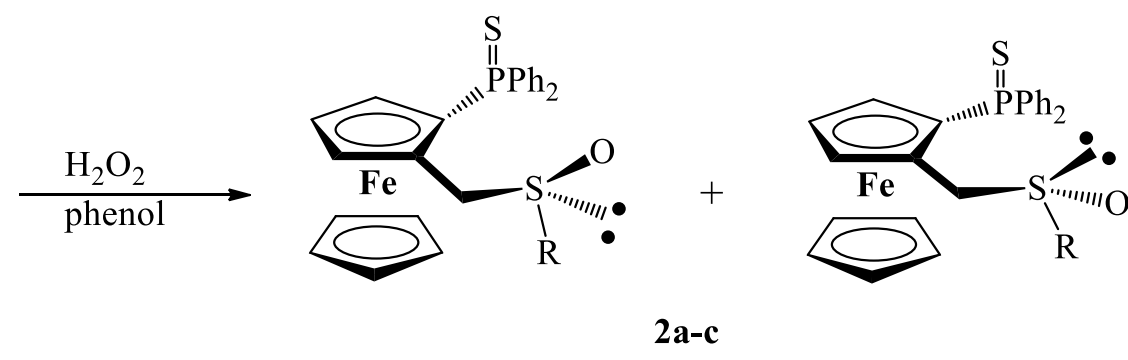

$\begin{array}{ll}\text { 2a } & 87 \%\left[\left(R_{\mathrm{Fc}}, R_{\mathrm{S}}\right)+\left(R_{\mathrm{Fc}}, S_{\mathrm{S}}\right)\right] \\ \text { 2b } & 87 \%\left[\left(R_{\mathrm{Fc}}, R_{\mathrm{S}}\right)+\left(R_{\mathrm{Fc}}, S_{\mathrm{S}}\right)\right] \\ \text { 2c } & 90 \%\left[\left(R_{\mathrm{Fc}}, R_{\mathrm{S}}\right)+\left(R_{\mathrm{Fc}}, S_{\mathrm{S}}\right)\right]\end{array}$

Scheme 2

As the thioetheroxidations wereperformed on ferrocene derivatives with thiophosphine group in order to prevent phosphorusoxidation, the next step was the phosphorus deprotectionin order to liberate the free phosphine group and generateferrocenylphosphinesulfoxidesthat can be used as chiral bidentate P-S ligands. This reaction was carried out using tris(dimethylamino)phosphine in refluxing toluene for 3 hours (Scheme 3). ${ }^{11 a}$ Longer reaction time gave partial deoxygenation of the sulfoxide group. Phosphine-sulfoxide compounds3a-cbeing air sensitive, their purification was accomplishedby column chromatography under argon with yields of isolated products from 65 to $93 \%$. Single crystals of $\left(S_{\mathbf{F c}}, S_{\mathbf{S}}\right) /\left(R_{\mathbf{F c}}, R_{\mathbf{S}}\right)-3 a$ were obtained by slow diffusion of pentane vapors into a dichloromethane solution, and the XRD structure is presented in Figure 4.The conformation of this unprotected liganddoesnot show significant differencesrelative tothe protected one $\left(\boldsymbol{S}_{\mathbf{F c}}, \boldsymbol{S}_{\mathbf{S}}\right) /\left(\boldsymbol{R}_{\mathbf{F c}}, \boldsymbol{R}_{\mathbf{S}}\right)-\mathbf{3 a}($ Table 1$)$. 


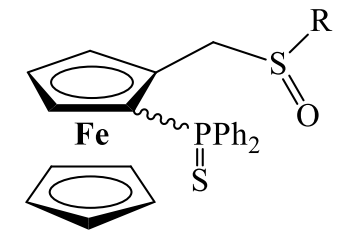

2a-c (Dia1 or Dia2)

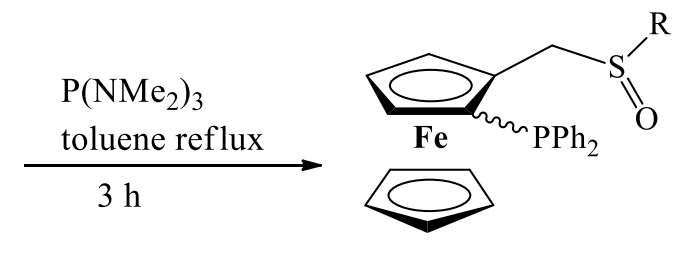

3
$\left(\mathbf{S}_{\mathbf{F c}}, \mathbf{R}_{\mathrm{S}}\right) /\left(\mathbf{R}_{\mathrm{Fc}}, \mathbf{S}_{\mathrm{S}}\right)-\mathbf{3 a}(93 \%)$

$\left(\mathbf{S}_{\mathbf{F c}}, \mathbf{S}_{\mathrm{S}}\right) /\left(\mathbf{R}_{\mathrm{Fc}}, \mathbf{R}_{\mathrm{S}}\right)-\mathbf{3 a}(85 \%)$

$\left(\mathbf{S}_{\mathrm{Fc}}, \mathbf{S}_{\mathrm{S}}\right) /\left(\mathbf{R}_{\mathrm{Fc}}, \mathbf{R}_{\mathrm{S}}\right)-3 \mathrm{~b}(66 \%)$

$\left(\mathbf{S}_{\mathbf{F c}}, \mathbf{R}_{\mathrm{S}}\right) /\left(\mathbf{R}_{\mathrm{Fc}}, \mathbf{S}_{\mathrm{S}}\right)-3 \mathbf{b}(65 \%)$

$\left(\mathbf{S}_{\mathrm{Fc}}, \mathbf{R}_{\mathrm{S}}\right) /\left(\mathbf{R}_{\mathrm{Fc}}, \mathbf{S}_{\mathrm{S}}\right)-3 \mathrm{c}(76 \%)$

$\left(\mathbf{S}_{\mathbf{F c}}, \mathbf{S}_{\mathrm{S}}\right) /\left(\mathbf{R}_{\mathrm{Fc}}, \mathbf{R}_{\mathrm{S}}\right)-3 \mathrm{c}(86 \%)$

\section{Scheme 3}

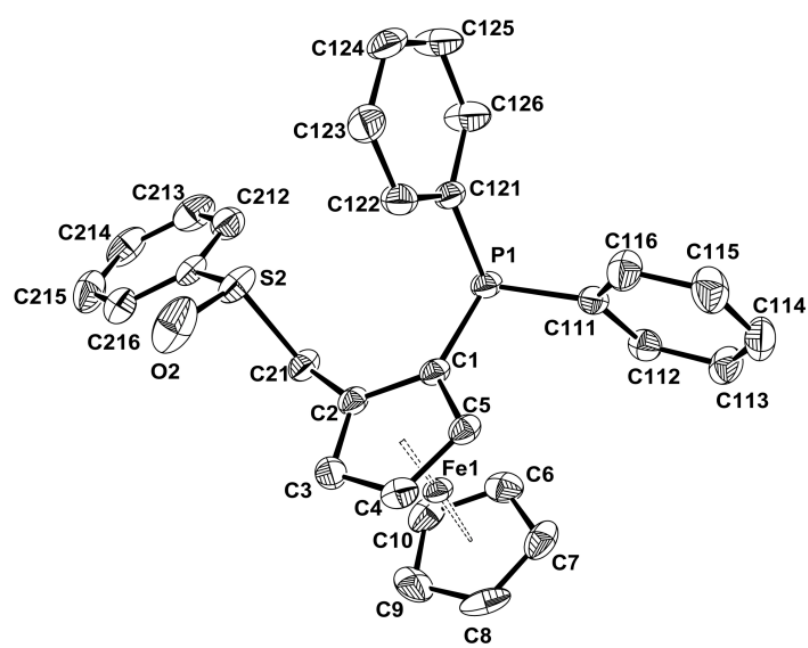

Figure 4.ORTEPview of the phosphine sulfoxyde3a-Dia2 $\left(S_{\mathrm{Fc}}, S_{\mathrm{S}}\right)$ with the atom labeling scheme. Ellipsoids are drawn at the $50 \%$ probability level. $\mathrm{H}$ atoms have been omitted for the sake of clarity.

Given that the $\mathrm{P}, \mathrm{SO}$ ligands could adoptdifferent coordination modes $\left(\kappa^{2}-P S, \kappa^{2}-P O\right.$, or $\left.\kappa^{1}-P\right),{ }^{17}$ wecarried out preliminary coordination chemistry investigations with platinum, byreactingthe phosphinesulfoxide ligand 3a (as the racemic diastereoisomericmixture, Dia1/Dia2 = 1/1) with the platinum precursor $\mathrm{PtCl}_{2}\left(\mathrm{CH}_{3} \mathrm{CN}\right)_{2}$. The ${ }^{31} \mathrm{P}\left\{{ }^{1} \mathrm{H}\right\}$ NMRanalysis of the final mixture shows the presence of two signals corresponding to two similar platinum complexes $\left(\delta 1=0.72 \mathrm{ppm}, \mathrm{J}_{\mathrm{P}-\mathrm{Pt}}=3338 \mathrm{~Hz}\right.$ and $\left.\delta 2=0.47 \mathrm{ppm}, \mathrm{J}_{\mathrm{P}-\mathrm{Pt}}=3444 \mathrm{~Hz}\right)$ in a $1: 1$ ratio. The ${ }^{1} \mathrm{~J}_{\mathrm{PtP}} \mathrm{alue}$ is characteristic of square planar complexes with cischlorine atoms. ${ }^{18} \mathrm{Crystals}$ of one complex were obtained by diffusion of pentane into a dichloromethane solution, and thus the coordination mode of the sulfoxide group as $\kappa^{2}-P S$ was determined from the XRD structure (See figure 5). 


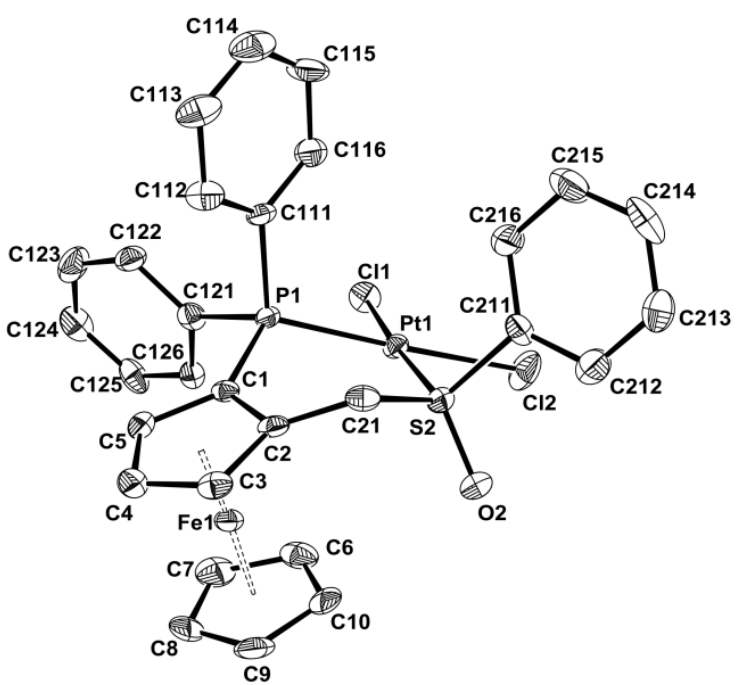

Figure 5.ORTEP view of platinum complex $\left(\boldsymbol{R}_{\mathbf{F c}}, \boldsymbol{R} \mathbf{s}\right)$-4awith the atom labeling scheme. The other enantiomer (not shown) is also present in the crystal.Ellipsoids are drawn at the 50\% probability level. $\mathrm{H}$ atoms have been omitted for the sake of clarity. Selected bond lengths ( $\AA$ ): Pt-P: 2,2350(12); Pt-S: 2.2291(12); Pt-Cl1(trans S): 2.2973(12); Pt-Cl2(transP): 2.3312(13); S-O: 1.459 .

The Pt atom is surrounded by theS2, P1, Cl1 and Cl2donor atoms in square planar coordination geometry. Itdeviatesfrom the least squares plane defined by these four atoms by only -0.0181(7) ^. The C1, C2, C21, S2, Pt1, P1 metallacycledisplays a boat conformation as indicated by the puckering parameters $\varphi=302.6(3)^{\circ}$ and $\theta=87.9(3) .{ }^{19}$ The S2 and $\mathrm{O} 2$ atoms are endo with respect to the Cp1 ring by -0.970(9) $\AA$ and -2.186(9) $\AA$ respectively. The coordination has induced a slight twist of the two Cp rings $\left(\tau=14.5(4)^{\circ}\right)$ when compared to the uncoordinated ligand which was roughly eclipsed with a twist angle of $4.7(2)^{\circ}$.

The crystallizedcompound corresponds to the racemic diastereomer with the $\left(R_{\mathrm{Fc}}, R_{\mathrm{S}}\right) /\left(S_{\mathrm{Fc}}, S_{\mathrm{S}}\right)$ configuration. Given the similarity of the NMR signals, we attribute to the second compound the structure $\mathbf{4 a}$ ' with the $\left(S_{\mathrm{Fc}}, R_{\mathrm{S}}\right) /\left(R_{\mathrm{Fc}}, S_{\mathrm{S}}\right)$ configuration (Scheme 4$)$.

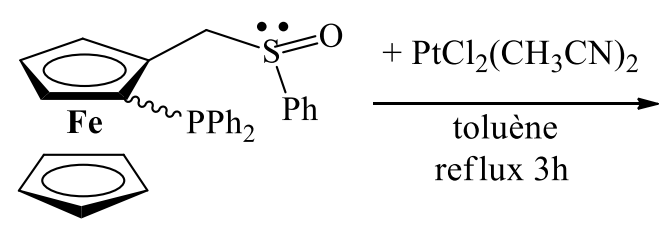

$3 \mathbf{a}$

$($ Dia $1+$ Dia 2)

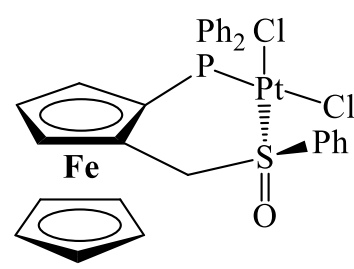

$4 a$

$\left(R_{\mathrm{Fc}}, R_{\mathrm{S}}\right) /\left(S_{\mathrm{Fc}}, S_{\mathrm{S}}\right)$

Scheme 4

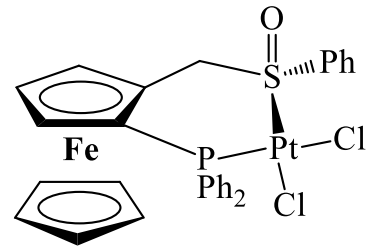

$4 \mathbf{a}^{\prime}$

$\left(S_{\mathrm{Fc}}, R_{\mathrm{S}}\right) /\left(R_{\mathrm{Fc}}, S_{\mathrm{S}}\right)$ 
The presence of two complexes in a 1:1 ratio in the product suggests that the two diastereomericferrocenyl phosphine-sulfoxide ligands do nothave significantly different reactivity with the platinum precursor. This might be surprising given that in the case of the correspondingferrocenyl phosphine thioethers, we observed a total discrimination between the two electron pairs for coordination with platinum, palladium ${ }^{20}$ or iridium. ${ }^{21}$ Our attempts to obtain complex $\mathbf{4 a}$ by oxidation of the sulfur atom in the coordination sphere of the corresponding phosphine-thioether platinum precursor $\left(\mathrm{Ph}_{2} \mathrm{P}-\mathrm{Fc}-\mathrm{CH}_{2} \mathrm{SPh}\right) \mathrm{PtCl}_{2}{ }^{20}$ were unsuccessful (conditions : mCPBA, THF, reflux, 20h). The oxidation reaction did not occur and the starting material was recovered quantitatively.

\section{Conclusion}

In conclusion, we have described the synthesis of new ferrocenyl derivatives thiophosphine-sulfoxide2a-c and phosphine-sulfoxide3a-c having aromatic or aliphatic substituents on the sulfur atom. These ligands possess planar chirality for theferrocenemoiety and central chirality at the sulfur atom and could be obtained as pure diastereoisomersin both racemic andenantiomerically pure form. Most of these compounds were fully characterized by XRD analysis allowingthe absolute configuration assignmentfor each stereoisomer.Preliminarycoordination studies of these ligands were carried out with platinum, demonstrating bidentate $\kappa^{2}-P S$ complexation in this case. Tests of enantiomerically pure ligands in various catalytic asymmetric reactions are in progress in our laboratories and will be presented in due course.

\section{Experimental}

\subsection{General}

All reactions were carried out under argon using standardSchlenkline techniques. Solvents were carefully dried by conventionalmethods and distilled under argon before use. The NMR spectra were recorded on a BrukerDRX-500Avanceinstrument with degased solvents. Elemental analyses were recorded on Perkin Elmer 2400 II. The mass spectrawererecordedon a Nermag R-10-10H spectrometer.

\subsection{2-thiodiphenylphosphino-(phenyl-sulfoxymethyl)ferrocene, $2 \mathrm{a}$.}

In a Schlenktube under argon were introduced $240 \mathrm{mg}(0.45 \mathrm{mmol})$ of racemic thioether $\left(R / S_{\mathrm{Fc}}\right)-1 \mathrm{a}$ and $500 \mathrm{mg}(5.4 \mathrm{mmol})$ of phenol. The mixture was heated to $35^{\circ} \mathrm{C}$, then $0.1 \mathrm{ml}$ of an aqueous solution of $\mathrm{H}_{2} \mathrm{O}_{2}(30 \%)$ were slowly added to the reaction medium. The reaction is highly exothermic and within minutes the solution turned black. After 5 minutes, $10 \mathrm{ml}$ of ethyl acetate, then $10 \mathrm{ml}$ of a saturated solution of $\mathrm{Na}_{2} \mathrm{SO}_{3}$, and finally $20 \mathrm{ml}$ of an aqueous solution of $\mathrm{NaOH}(10 \%)$ were added. The phases were separated and the aqueous phase was 
extracted with three portions $(10 \mathrm{ml})$ of ethyl acetate. The combined organic layers were washed with water, dried over $\mathrm{MgSO}_{4}$ and then the solvent was removed by evaporation under reduced pressure.Compound 2awas recovered as an orange solid (yield: $226 \mathrm{mg}$, 92\%). The two diastereomers were separated by chromatography on silica usinga mixture of ether and dichloromethane (1/1) as eluent to give $100 \mathrm{mg}$ of $\mathbf{2 a - D i a 1 ,}\left(\boldsymbol{R}_{\mathbf{F c}}, \boldsymbol{S} \mathbf{s}\right) /\left(\boldsymbol{S}_{\mathbf{F c}}, \boldsymbol{R} \mathbf{s}\right)-\mathbf{2 a}$ (yield: $41 \%)$ and $108 \mathrm{mg}$ of 2a-Dia2, $\left(\boldsymbol{R}_{\mathbf{F c}}, \boldsymbol{R} \mathbf{S}\right) /\left(\boldsymbol{S}_{\mathbf{F c}}, \mathbf{S}_{\mathbf{S}}\right)-\mathbf{2 a}$ (yield: $\left.44 \%\right) . \mathrm{MS}\left(\mathrm{DCI}, \mathrm{NH}_{3}\right): \mathrm{m} / \mathrm{z}=541$ $(\mathrm{M}+1,20 \%) ; 558\left(\mathrm{M}+\mathrm{NH}_{4}, 100 \%\right)$.

$\underline{\left(\boldsymbol{R}_{\mathbf{F c}}, S_{\mathbf{s}}\right) /\left(\boldsymbol{S}_{\mathbf{F c}}, \boldsymbol{R} \mathbf{s}\right)-\mathbf{2 a}}:{ }^{1} \mathrm{H}$ NMR $\left(\delta, 500 \mathrm{MHz}, \mathrm{CDCl}_{3}\right): 7.86(2 \mathrm{H}, \mathrm{m}, \mathrm{Ph}) ; 7.65-7.40(13 \mathrm{H}, \mathrm{m}$, $\mathrm{Ph}) ; 4.48(1 \mathrm{H}$, broad s, substCp); $4.44(5 \mathrm{H}, \mathrm{s}, \mathrm{Cp}) ; 4.35(1 \mathrm{H}$. broad s, substCp); $4.32(1 \mathrm{H}$, $\left.\mathrm{d}(\mathrm{AB}), \mathrm{J}_{\mathrm{HH}}=13 \mathrm{~Hz}, \underline{\mathrm{H}}_{2} \mathrm{Cp}\right) ; 4.30\left(1 \mathrm{H}, \mathrm{d}(\mathrm{AB}), \mathrm{J}_{\mathrm{HH}}=13 \mathrm{~Hz}, \underline{\mathrm{H}}_{2} \mathrm{Cp}\right) ; 3.87(1 \mathrm{H}$, broad $\mathrm{s}$, substCp). ${ }^{13} \mathrm{C}\left\{{ }^{1} \mathrm{H}\right\}$ NMR $\left(\delta, 125.8 \mathrm{MHz}, \mathrm{CDCl}_{3}\right): 144.5$ (s, quatSPh); $134.8\left(\mathrm{~d}, \mathrm{~J}_{\mathrm{CP}}=87 \mathrm{~Hz}\right.$, quat $\left.\mathrm{PPh}_{2}\right) ; 133.1\left(\mathrm{~d}, \mathrm{~J}_{\mathrm{CP}}=86 \mathrm{~Hz}\right.$, quat $\left.\mathrm{PPh}_{2}\right) ; 132.5\left(\mathrm{~d}, \mathrm{~J}_{\mathrm{CP}}=11 \mathrm{~Hz}, \mathrm{PPh}_{2}\right) ; 132.3\left(\mathrm{~d}, \mathrm{~J}_{\mathrm{CP}}=11\right.$ $\left.\mathrm{Hz}, \mathrm{PPh}_{2}\right) ; 131.96$ (s, $\left.\mathrm{PPh}_{2}\right) ; 131.9\left(\mathrm{~s}, \mathrm{PPh}_{2}\right) ; 131.3$ (s, SPh); 129.4 (s, SPh); $128.9\left(\mathrm{~d}, \mathrm{~J}_{\mathrm{CP}}=13\right.$ $\left.\mathrm{Hz}, \mathrm{PPh}_{2}\right) ; 128.6\left(\mathrm{~d}, \mathrm{~J}_{\mathrm{CP}}=13 \mathrm{~Hz}, \mathrm{PPh}_{2}\right) ; 124.8(\mathrm{~s}, \mathrm{SPh}) ; 83.1$ (d, $\mathrm{J}_{\mathrm{CP}}=12 \mathrm{~Hz}$, quatCp); 75.8 $\left(\mathrm{d}, \mathrm{J}_{\mathrm{CP}}=95 \mathrm{~Hz}\right.$, quatCp); $75.4\left(\mathrm{~d}, \mathrm{~J}_{\mathrm{CP}}=9 \mathrm{~Hz}\right.$, substCp); $74.6\left(\mathrm{~d}, \mathrm{~J}_{\mathrm{CP}}=12 \mathrm{~Hz}\right.$, substCp); 71.6 (s, $\mathrm{Cp}) ; 70.4\left(\mathrm{~d}, \mathrm{~J}_{\mathrm{CP}}=10 \mathrm{~Hz}\right.$, substCp); $58.0\left(\mathrm{~s}, \underline{\mathrm{CH}}_{2} \mathrm{Cp}\right) .{ }^{31} \mathrm{P}\left\{{ }^{1} \mathrm{H}\right\} \mathrm{NMR}(\delta, 202.5 \mathrm{MHz}$. $\mathrm{CDCl}_{3}$ ):43.5. Elemental analysis: $\mathrm{C}_{2} \mathrm{H}_{25} \mathrm{FePS}_{2} \mathrm{O} ; \% \mathrm{C}$ (calcd 64.44, found 62.84), \% $\mathrm{H}$ (calcd 4.63, found 5.03).

$\underline{(\boldsymbol{R} \mathbf{F c}}, \underline{R} \mathbf{s}) /\left(\boldsymbol{S}_{\mathbf{F c}}, \underline{S} \mathbf{s}\right)-\mathbf{2 a}:{ }^{1} \mathrm{H}$ NMR $\left(\delta, 500 \mathrm{MHz}, \mathrm{CDCl}_{3}\right): 7.89(2 \mathrm{H}, \mathrm{m}, \mathrm{Ph}) ; 7.87(2 \mathrm{H}, \mathrm{m}, \mathrm{Ph}) ; 7.6-$ $7.40(11 \mathrm{H}, \mathrm{m}, \mathrm{Ph}) ; 5.34\left(1 \mathrm{H}, \mathrm{d}(\mathrm{AB}), \mathrm{J}_{\mathrm{HH}}=13 \mathrm{~Hz}, \underline{C}_{2} \mathrm{Cp}\right) ; 4.85(1 \mathrm{H}, \mathrm{m}, \operatorname{substCp}) ; 4.49(1 \mathrm{H}$, m, substCp); $4.30(5 \mathrm{H}, \mathrm{s}, \mathrm{Cp}) ; 3.87\left(1 \mathrm{H}, \mathrm{m}\right.$, substCp); $3.61\left(1 \mathrm{H}, \mathrm{d}(\mathrm{AB}), \mathrm{J}_{\mathrm{HH}}=13 \mathrm{~Hz}\right.$, $\left.\mathrm{C}_{2} \mathrm{Cp}\right) .{ }^{13} \mathrm{C}\left\{{ }^{1} \mathrm{H}\right\} \operatorname{NMR}\left(\delta, 125.8 \mathrm{MHz}, \mathrm{CDCl}_{3}\right): 134.9\left(\mathrm{~d}, \mathrm{~J}_{\mathrm{CP}}=87 \mathrm{~Hz}\right.$, quat $\left.\mathrm{PPh}_{2}\right) ; 133.2(\mathrm{~d}$, $\mathrm{J}_{\mathrm{CP}}=87 \mathrm{~Hz}$, quat $\left.\mathrm{PPh}_{2}\right) ; 132.7\left(\mathrm{~d}, \mathrm{~J}_{\mathrm{CP}}=11 \mathrm{~Hz}, \mathrm{PPh}_{2}\right) ; 132.4\left(\mathrm{~d}, \mathrm{~J}_{\mathrm{CP}}=11 \mathrm{~Hz}, \mathrm{PPh}_{2}\right) ; 132.1(\mathrm{~d}$, $\left.\mathrm{J}_{\mathrm{CP}}=3 \mathrm{~Hz}, \mathrm{PPh}_{2}\right) ; 132.0\left(\mathrm{~d}, \mathrm{~J}_{\mathrm{CP}}=3 \mathrm{~Hz}, \mathrm{PPh}_{2}\right) ; 130.9(\mathrm{~s}, \mathrm{SPh}) ; 129.5(\mathrm{~s}, \mathrm{SPh}) ; 129.4(\mathrm{~s}$, quatSPh); $128.8\left(\mathrm{~d}, \mathrm{~J}_{\mathrm{CP}}=13 \mathrm{~Hz}, \mathrm{PPh}_{2}\right) ; 128.6\left(\mathrm{~d}, \mathrm{~J}_{\mathrm{CP}}=13 \mathrm{~Hz}, \mathrm{PPh}_{2}\right) ; 123.9(\mathrm{~s}, \mathrm{SPh}) ; 83.0(\mathrm{~d}$, $\mathrm{J}_{\mathrm{CP}}=12 \mathrm{~Hz}$, quatCp); $76.2\left(\mathrm{~d}, \mathrm{~J}_{\mathrm{CP}}=9 \mathrm{~Hz}\right.$, substCp); 75.8 (d, $\mathrm{J}_{\mathrm{CP}}=12 \mathrm{~Hz}$, substCp); 74.8 (d, $\mathrm{J}_{\mathrm{CP}}=95 \mathrm{~Hz}$, quatCp); $71.4(\mathrm{~s}, \mathrm{Cp}) ; 71.1\left(\mathrm{~d}, \mathrm{~J}_{\mathrm{CP}}=10 \mathrm{~Hz}\right.$, substCp); $61.1\left(\mathrm{~s}, \underline{\mathrm{C}} \mathrm{H}_{2} \mathrm{Cp}\right) .{ }^{31} \mathrm{P}\left\{{ }^{1} \mathrm{H}\right\}$ NMR ( $\left.\delta, 202.5 \mathrm{MHz}, \mathrm{CDCl}_{3}\right): 43.9$; Elemental analysis: $\mathrm{C}_{29} \mathrm{H}_{25} \mathrm{FePS}_{2} \mathrm{O}$; \%C (calcd 64.44, found 64.12), \% $\mathrm{H}$ (calcd 4.63, found 4.32).

\subsection{2-thiodiphenylphosphino-(ethyl-sulfoxymethyl)-ferrocene $2 \mathrm{~b}$}

Following the same procedure described abovefor the synthesis of $\mathbf{2 a}$, the reaction was realized starting from $250 \mathrm{mg}(0.52 \mathrm{mmol})$ of racemic thioether $(R / S)_{\mathrm{Fc}}-\mathbf{1 b}, 500 \mathrm{mg}$ of phenol 
and $0.10 \mathrm{ml}$ of an aqueous solution of $\mathrm{H}_{2} \mathrm{O}_{2}(30 \%)$. 2bwas recovered as an orange solid (yield: $241 \mathrm{mg}, 93 \%$ ). The two diastereomers were separated by chromatography on silica usinga mixture of ether and dichloromethane (1/1) as eluent to give $114 \mathrm{mg}$ of $\mathbf{2 b}$-Dia1,

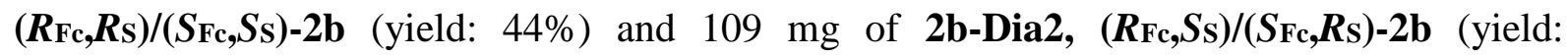
42\%).MS (DCI, $\left.\mathrm{NH}_{3}\right): \mathrm{m} / \mathrm{z}=493(\mathrm{M}+1,45 \%) ; 510\left(\mathrm{M}+\mathrm{NH}_{4}, 100 \%\right)$.

$\underline{\left(\boldsymbol{R}_{\mathbf{F c}}, \underline{R} \mathbf{S}\right) /\left(\boldsymbol{S}_{\mathbf{F c}}, S_{\mathbf{S}}\right)-\mathbf{2 b}}:{ }^{1} \mathrm{H}$ NMR $\left(\delta, 500 \mathrm{MHz} . \mathrm{CDCl}_{3}\right): 7.85-7.81\left(2 \mathrm{H}, \mathrm{m}, \mathrm{PPh}_{2}\right) ; 7.66-7.47(6 \mathrm{H}$, m, $\left.\mathrm{PPh}_{2}\right) ; 7.43\left(2 \mathrm{H}\right.$, broad s, $\left.\mathrm{PPh}_{2}\right) ; 4.90(1 \mathrm{H}, \mathrm{s}$, subst $\mathrm{Cp}) ; 4.47(1 \mathrm{H}, \mathrm{s}$, subst $\mathrm{Cp}) ; 4.36$ (5H, s, $\mathrm{Cp})$; 4.45-4.28 (2H, m, $\left.\underline{\mathrm{H}}_{2} \mathrm{Cp}\right) ; 3.87(1 \mathrm{H}$, s, subst $\mathrm{Cp}) ; 2.35\left(2 \mathrm{H}\right.$, broad s, $\left.\mathrm{C}_{2} \mathrm{CH}_{3}\right) ; 1.1(3 \mathrm{H}$, broad s, $\left.\mathrm{CH}_{2} \underline{\mathrm{C}}_{3}\right) .{ }^{13} \mathrm{C}\left\{{ }^{1} \mathrm{H}\right\} \mathrm{NMR}\left(\delta, 125.8 \mathrm{MHz}^{\mathrm{CDCl}} \mathrm{CD}_{3}\right): 133.9\left(\mathrm{~d}, \mathrm{~J}_{\mathrm{CP}}=87 \mathrm{~Hz}\right.$, quat $\left.\mathrm{PPh}_{2}\right)$; $132.1\left(\mathrm{~d}, \mathrm{~J}_{\mathrm{CP}}=86 \mathrm{~Hz}\right.$, quat $\left.\mathrm{PPh}_{2}\right) ; 132.0\left(\mathrm{~d}, \mathrm{~J}_{\mathrm{CP}}=11 \mathrm{~Hz}, \mathrm{PPh}_{2}\right) ; 131.9\left(\mathrm{~s}, \mathrm{PPh}_{2}\right) ; 131.8($ broad $\left.\mathrm{s}, \mathrm{PPh}_{2}\right) ; 128.7\left(\mathrm{~d}, \mathrm{~J}_{\mathrm{CP}}=12 \mathrm{~Hz}, \mathrm{PPh}_{2}\right) ; 128.5\left(\mathrm{~d}, \mathrm{~J}_{\mathrm{CP}}=12 \mathrm{~Hz}, \mathrm{PPh}_{2}\right) ; 82.0\left(\mathrm{~d}, \mathrm{~J}_{\mathrm{CP}}=12 \mathrm{~Hz}\right.$, quat Cp); 74.8 (s, subst Cp); $74.7\left(\mathrm{~d}, \mathrm{~J}_{\mathrm{CP}}=12 \mathrm{~Hz}\right.$, subst $\left.\mathrm{Cp}\right) ; 74.0\left(\mathrm{~d}, \mathrm{~J}_{\mathrm{CP}}=96 \mathrm{~Hz}\right.$, quat Cp); 71.3 (s, Cp); 70.8 (d, J $\mathrm{CP}_{\mathrm{CP}} 10 \mathrm{~Hz}$, subst Cp); 50.6 (s, $\left.\underline{\mathrm{CH}}_{2} \mathrm{Cp}\right) ; 44.7\left(\mathrm{~s}, \underline{\mathrm{CH}}_{2} \mathrm{CH}_{3}\right) ; 7.6\left(\mathrm{~s}, \mathrm{CH}_{2} \underline{\mathrm{CH}}_{3}\right)$. ${ }^{31} \mathrm{P}\left\{{ }^{1} \mathrm{H}\right\} \mathrm{NMR}\left(\delta, 202.5 \mathrm{MHz}, \mathrm{CDCl}_{3}\right)$ : 41.06. Elemental analysis: $\mathrm{C}_{25} \mathrm{H}_{25} \mathrm{FePS}_{2} \mathrm{O} ; \% \mathrm{C}$ (calcd 60.98 , found 61.08 ), \% $\mathrm{H}$ (calcd 5.08, found 5.17).

$\underline{\left(\boldsymbol{R}_{\mathbf{F c}}, \boldsymbol{S}_{\mathbf{S}}\right) /\left(\boldsymbol{S}_{\mathrm{Fc}}, \boldsymbol{R} \mathbf{S}\right)-\mathbf{2 b}}:{ }^{1} \mathrm{H} \mathrm{NMR}\left(\delta, 500 \mathrm{MHz} . \mathrm{CDCl}_{3}\right): 7.83-7.79\left(2 \mathrm{H}, \mathrm{m}, \mathrm{PPh}_{2}\right) ; 7.59-7.48(6 \mathrm{H}$, m, $\left.\mathrm{PPh}_{2}\right) ; 7.41\left(2 \mathrm{H}\right.$, broad s, $\left.\mathrm{PPh}_{2}\right) ; 5.26\left(1 \mathrm{H}, \mathrm{d}(\mathrm{AB}), \mathrm{J}_{\mathrm{HH}}=13 \mathrm{~Hz}, \mathrm{CH}_{2} \mathrm{Cp}\right) ; 4.81(1 \mathrm{H}, \mathrm{s}$, subst $\mathrm{Cp}) ; 4.47\left(1 \mathrm{H}, \mathrm{s}\right.$, subst Cp); $4.32(5 \mathrm{H}, \mathrm{s}, \mathrm{Cp}) ; 3.82(1 \mathrm{H}, \mathrm{s}$, subst $\mathrm{Cp}) ; 3.53\left(1 \mathrm{H}, \mathrm{d}(\mathrm{AB}), \mathrm{J}_{\mathrm{HH}}=\right.$ $\left.13 \mathrm{~Hz}, \underline{\mathrm{CH}}_{2} \mathrm{Cp}\right) ; 2.65\left(2 \mathrm{H}, \mathrm{q}, \mathrm{J}_{\mathrm{HH}}=7 \mathrm{~Hz}, \mathrm{C}_{2} \mathrm{CH}_{3}\right) ; 1.5\left(3 \mathrm{H}\right.$, broad s, $\left.\mathrm{CH}_{2} \mathrm{CH}_{3}\right) .{ }^{13} \mathrm{C}\left\{{ }^{1} \mathrm{H}\right\}$ $\operatorname{NMR}\left(\delta, 125.8 \mathrm{MHz}, \mathrm{CDCl}_{3}\right): 134.1\left(\mathrm{~d}, \mathrm{~J}_{\mathrm{CP}}=88 \mathrm{~Hz}\right.$, quat $\left.\mathrm{PPh}_{2}\right) ; 132.3\left(\mathrm{~d}, \mathrm{~J}_{\mathrm{CP}}=88 \mathrm{~Hz}\right.$, quat $\left.\mathrm{PPh}_{2}\right) ; 132.0\left(\mathrm{~d}, \mathrm{~J}_{\mathrm{CP}}=11 \mathrm{~Hz}, \mathrm{PPh}_{2}\right) ; 131.9\left(\right.$ broad s, $\left.\mathrm{PPh}_{2}\right) ; 128.6\left(\mathrm{~d}, \mathrm{~J}_{\mathrm{CP}}=12 \mathrm{~Hz}, \mathrm{PPh}_{2}\right) ; 128.5$ $\left(\mathrm{d}, \mathrm{J}_{\mathrm{CP}}=13 \mathrm{~Hz}, \mathrm{PPh}_{2}\right) ; 82.5\left(\mathrm{~d}, \mathrm{~J}_{\mathrm{CP}}=10 \mathrm{~Hz}\right.$, quat $\left.\mathrm{Cp}\right) ; 75.8\left(\mathrm{~d}, \mathrm{~J}_{\mathrm{CP}}=9 \mathrm{~Hz}\right.$, subst $\left.\mathrm{Cp}\right) ; 75.5(\mathrm{~d}$, $\mathrm{J}_{\mathrm{CP}}=12 \mathrm{~Hz}$, subst Cp); $73.1\left(\mathrm{~d}, \mathrm{~J}_{\mathrm{CP}}=95 \mathrm{~Hz}\right.$,quat Cp); 71.2 (s, Cp); 71.1 (s, subst Cp); 53.4 (s, $\left.\underline{\mathrm{C}}_{2} \mathrm{Cp}\right) ; 46.7 \quad\left(\mathrm{~s}, \quad \underline{\mathrm{CH}}_{2} \mathrm{CH}_{3}\right) ; 7.1 \quad\left(\mathrm{~s}, \quad \mathrm{CH}_{2} \underline{\mathrm{CH}}_{3}\right) . \quad{ }^{31} \mathrm{P}\left\{{ }^{1} \mathrm{H}\right\} \quad \mathrm{NMR} \quad(\delta, \quad 202.5 \mathrm{MHz}$, $\mathrm{CDCl}_{3}$ ):41.6.Elemental analysis: $\mathrm{C}_{25} \mathrm{H}_{25} \mathrm{FePS}_{2} \mathrm{O} ; \% \mathrm{C}$ (calcd 60.98, found 60.98), \% $\mathrm{H}$ (calcd 5.08 , found 4.83$)$.

\subsection{2-thiodiphenylphosphino-(tert-butyl-sulfoxymethyl)-ferrocene2c}

Following the same procedure described abovefor the synthesis of $\mathbf{2 a}$, the reaction was carried outstarting from $250 \mathrm{mg}(0.496 \mathrm{mmol})$ of racemic thioether $(R / S)_{\mathrm{Fc}}-1 \mathrm{c}, 500 \mathrm{mg}$ of phenol and $0.10 \mathrm{ml}$ of an aqueous solution of $\mathrm{H}_{2} \mathrm{O}_{2}(30 \%)$. 2cwas recovered as an orange solid (yield: 237 $\mathrm{mg}, 92 \%)$. The two diastereomers were separated by chromatography on silica usinga mixture

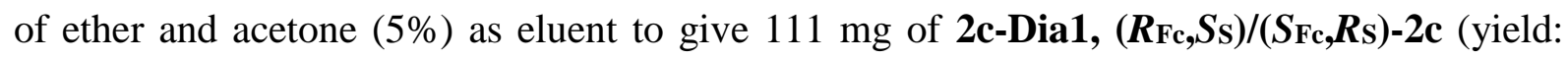


43\%) and $103 \mathrm{mg}$ of $\mathbf{2 c - D i a 2 ,}\left(\boldsymbol{R}_{\mathrm{Fc}}, \boldsymbol{R} \mathbf{S}\right) /\left(\boldsymbol{S}_{\mathrm{Fc}}, \boldsymbol{S}_{\mathbf{S}}\right)-\mathbf{2 c}($ yield: $40 \%) . \mathrm{MS}\left(\mathrm{DCI}, \mathrm{NH}_{3}\right): \mathrm{m} / \mathrm{z}=521$ $(\mathrm{M}+1,100 \%) ; 538\left(\mathrm{M}+\mathrm{NH}_{4}, 80 \%\right)$.

$\left(\boldsymbol{R}_{\mathbf{F c}}, S_{\mathbf{S}}\right) /\left(\boldsymbol{S}_{\mathbf{F c}}, \boldsymbol{R}_{\mathbf{S}}\right)-2 \mathrm{c}:{ }^{1} \mathrm{H}$ NMR $\left(\delta, 500 \mathrm{MHz}, \mathrm{CDCl}_{3}\right): 7.8\left(2 \mathrm{H}, \mathrm{m}, \mathrm{PPh}_{2}\right) ; 7.58-7.47(6 \mathrm{H}, \mathrm{m}$, $\left.\mathrm{PPh}_{2}\right) ; 7.40\left(2 \mathrm{H}, \mathrm{m}, \mathrm{PPh}_{2}\right) ; 5.07(1 \mathrm{H}$, broad s, subst $\mathrm{Cp}) ; 4.47\left(1 \mathrm{H}\right.$, broad s, $\left.\mathrm{C}_{2} \mathrm{Cp}\right) ; 4.41(5 \mathrm{H}$, s, Cp) ; 4.41(1H, s, subst Cp); $3.78\left(1 \mathrm{H}\right.$, s, subst Cp); $3.05\left(1 \mathrm{H}, \mathrm{d}(\mathrm{AB}), \mathrm{J}_{\mathrm{HH}}=14 \mathrm{~Hz}, \underline{\mathrm{C}}_{2} \mathrm{Cp}\right)$; $1.32\left(9 \mathrm{H}, \mathrm{s}, \mathrm{C}\left(\mathrm{CH}_{3}\right)_{3}\right) .{ }^{13} \mathrm{C}\left\{{ }^{1} \mathrm{H}\right\} \mathrm{NMR}\left(\delta, 125.8 \mathrm{MHz}, \mathrm{CDCl}_{3}\right): 134.6\left(\mathrm{~d}, \mathrm{~J}_{\mathrm{CP}}=87 \mathrm{~Hz}\right.$, quat $\left.\mathrm{PPh}_{2}\right) ; 132.4\left(\mathrm{~d}, \mathrm{~J}_{\mathrm{CP}}=86 \mathrm{~Hz}\right.$, quat $\left.\mathrm{PPh}_{2}\right) ; 131.9\left(\mathrm{~d}, \mathrm{~J}_{\mathrm{CP}}=11 \mathrm{~Hz}, \mathrm{PPh}_{2}\right) ; 131.7\left(\mathrm{~d}, \mathrm{~J}_{\mathrm{CP}}=11 \mathrm{~Hz}\right.$, $\left.\mathrm{PPh}_{2}\right) ; 131.6\left(\mathrm{~d}, \mathrm{~J}_{\mathrm{CP}}=3 \mathrm{~Hz}, \mathrm{PPh}_{2}\right) ; 131.5\left(\mathrm{~d}, \mathrm{~J}_{\mathrm{CP}}=3 \mathrm{~Hz}, \mathrm{PPh}_{2}\right) ; 128.6\left(\mathrm{~d}, \mathrm{~J}_{\mathrm{CP}}=13 \mathrm{~Hz}, \mathrm{PPh}_{2}\right)$; $128.2\left(\mathrm{~d}, \mathrm{~J}_{\mathrm{CP}}=13 \mathrm{~Hz}, \mathrm{PPh}_{2}\right) ; 85.2\left(\mathrm{~d}, \mathrm{~J}_{\mathrm{CP}}=12 \mathrm{~Hz}\right.$, quat $\left.\mathrm{Cp}\right) ; 74.9\left(\mathrm{~d}, \mathrm{~J}_{\mathrm{CP}}=96 \mathrm{~Hz}\right.$, quat $\left.\mathrm{Cp}\right)$; $73.8\left(\mathrm{~d}, \mathrm{~J}_{\mathrm{CP}}=12 \mathrm{~Hz}\right.$, subst Cp); $73.6\left(\mathrm{~d}, \mathrm{~J}_{\mathrm{CP}}=19 \mathrm{~Hz}\right.$, subst Cp); $71.1(\mathrm{~s}, \mathrm{Cp}) ; 70.0\left(\mathrm{~d}, \mathrm{~J}_{\mathrm{CP}}=9\right.$ $\mathrm{Hz}$, subst $\mathrm{Cp}$ ); 54.2 (broad s, $\left.\underline{\mathrm{C}}\left(\mathrm{CH}_{3}\right)_{3}\right) ; 44.9$ (s, $\left.\underline{\mathrm{CH}}_{2} \mathrm{Cp}\right) ; 23.1\left(\mathrm{~s}, \mathrm{C}\left(\underline{\mathrm{CH}}_{3}\right)_{3}\right) .{ }^{31} \mathrm{P}\left\{{ }^{1} \mathrm{H}\right\} \mathrm{NMR}$ $\left(\delta, 202.5 \mathrm{MHz}, \mathrm{CDCl}_{3}\right)$ : 41.8. Elemental analysis: $\mathrm{C}_{27} \mathrm{H}_{29} \mathrm{FePS} 2 \mathrm{O} ; \% \mathrm{C}$ (calcd 62.31, found 62.16), \% H (calcd 5.58, found 5.54).

$\left(\boldsymbol{R}_{\mathbf{F c}}, \boldsymbol{R} \mathbf{s}\right) /\left(\boldsymbol{S}_{\mathbf{F c}}, \boldsymbol{S}_{\mathbf{S}}\right)-2 \mathbf{c}:{ }^{1} \mathrm{H}$ NMR $\left(\delta, 500 \mathrm{MHz}, \mathrm{CDCl}_{3}\right): 7.83\left(1 \mathrm{H}, \mathrm{dd}, \mathrm{J}_{\mathrm{HH}}=2 \mathrm{~Hz}, \mathrm{~J}_{\mathrm{HP}}=14 \mathrm{~Hz}, \mathrm{~m}\right.$ $\left.\mathrm{C}_{6} \underline{\mathrm{H}}_{5}\right) ; 7.82\left(1 \mathrm{H}, \mathrm{dd}, \mathrm{J}_{\mathrm{HH}}=2 \mathrm{~Hz}, \mathrm{~J}_{\mathrm{HP}}=15 \mathrm{~Hz}, \mathrm{~m} \mathrm{C}_{6} \underline{\mathrm{H}}_{5}\right) ; 7.66\left(1 \mathrm{H}, \mathrm{dd}, \mathrm{J}_{\mathrm{HH}}=2 \mathrm{~Hz}, \mathrm{~J}_{\mathrm{HP}}=15 \mathrm{~Hz}\right.$, $\left.\mathrm{m} \mathrm{C}_{6} \underline{\mathrm{H}}_{5}\right) ; 7.65\left(1 \mathrm{H}, \mathrm{dd}, \mathrm{J}_{\mathrm{HH}}=2 \mathrm{~Hz}, \mathrm{~J}_{\mathrm{HP}}=15 \mathrm{~Hz}, \mathrm{~m} \mathrm{C}_{6} \underline{\mathrm{H}}_{5}\right) ; 7.58-7.53\left(1 \mathrm{H}, \mathrm{m}, \mathrm{p} \mathrm{C} \mathrm{C}_{6} \underline{\mathrm{H}}_{5}\right) ; 7.52-$ $7.45\left(3 \mathrm{H}, \mathrm{m}, \mathrm{p}+2 \mathrm{o}_{6} \underline{\mathrm{H}}_{5}\right) ; 7.42-7.37\left(2 \mathrm{H}, \mathrm{m}, 2 \mathrm{o} \mathrm{C}_{6} \underline{\mathrm{H}}_{5}\right) ; 5.09\left(1 \mathrm{H}, \mathrm{d}(\mathrm{AB}) . \mathrm{J}_{\mathrm{HH}}=13 \mathrm{~Hz}\right.$, $\left.\mathrm{C}_{2} \mathrm{Cp}\right) ; 4.89$ (1H, m, subst Cp); 4.43 (1H, m, subst Cp); 4.37 (5H, s, Cp); 3.75 (1H, m, subst $\mathrm{Cp}) ; 3.28\left(1 \mathrm{H}, \mathrm{d}(\mathrm{AB}), \mathrm{J}_{\mathrm{HH}}=13 \mathrm{~Hz}, \underline{\mathrm{C}}_{2} \mathrm{Cp}\right) ; 1.04\left(9 \mathrm{H}, \mathrm{s}, \mathrm{C}\left(\mathrm{C}_{\mathrm{H}_{3}}\right)_{3}\right) .{ }^{13} \mathrm{C}\left\{{ }^{1} \mathrm{H}\right\} \mathrm{NMR}(\delta, 125.8$ MHz. $\left.\mathrm{CDCl}_{3}\right): 134.02\left(\mathrm{~d}, \mathrm{~J}_{\mathrm{CP}}=87 \mathrm{~Hz}\right.$, quat $\left.\mathrm{PPh}_{2}\right) ; 132.87\left(\mathrm{~d}, \mathrm{~J}_{\mathrm{CP}}=82 \mathrm{~Hz}\right.$, quat $\left.\mathrm{PPh}_{2}\right) ; 132.53$ $\left(\mathrm{d}, \mathrm{J}_{\mathrm{CP}}=10 \mathrm{~Hz}, \mathrm{~m} \mathrm{PPh}_{2}\right) ; 131.97\left(\mathrm{~d}, \mathrm{~J}_{\mathrm{CP}}=10 \mathrm{~Hz}, \mathrm{mPPh}_{2}\right) ; 131.50\left(\mathrm{~d}, \mathrm{~J}_{\mathrm{CP}}=3 \mathrm{~Hz}, \mathrm{pPPh}_{2}\right)$; $131.45\left(\mathrm{~d}, \mathrm{~J}_{\mathrm{CP}}=3 \mathrm{~Hz}, \mathrm{p} \mathrm{PPh}_{2}\right) ; 128.23\left(\mathrm{~d}, \mathrm{~J}_{\mathrm{CP}}=4 \mathrm{~Hz}, \mathrm{o} \mathrm{PPh}_{2}\right) ; 128.13$ (d. $\left.\mathrm{J}_{\mathrm{CP}}=4 \mathrm{~Hz}, \mathrm{o} \mathrm{PPh}_{2}\right)$; $83.50\left(\mathrm{~d}, \mathrm{~J}_{\mathrm{CP}}=12 \mathrm{~Hz}\right.$, quat Cp); $75.20\left(\mathrm{~d}, \mathrm{~J}_{\mathrm{CP}}=9 \mathrm{~Hz}\right.$, subst $\left.\mathrm{Cp}\right) ; 75.07$ (d, $\mathrm{J}_{\mathrm{CP}}=13 \mathrm{~Hz}$, subst $\mathrm{Cp}$ ); 74.47 (d, $\mathrm{J}_{\mathrm{CP}}=95 \mathrm{~Hz}$, quat Cp); 71.04 (s, Cp); 70.41 (d, $\mathrm{J}_{\mathrm{CP}}=10 \mathrm{~Hz}$, subst Cp); 53.23 (s, $\left.\underline{\mathrm{C}}\left(\mathrm{CH}_{3}\right)_{3}\right) ; \quad 47.84 \quad\left(\mathrm{~s}, \quad \underline{\mathrm{CH}_{2} \mathrm{Cp}}\right) ; 22.75 \quad$ (s, $\left.\quad \mathrm{C}\left(\underline{\mathrm{CH}}_{3}\right)_{3}\right) . \quad{ }^{31} \mathrm{P}\left\{{ }^{1} \mathrm{H}\right\} \quad \mathrm{NMR} \quad(\delta, 202.5 \quad \mathrm{MHz}$. $\mathrm{CDCl}_{3}$ ):41.4.Elemental analysis: $\mathrm{C}_{27} \mathrm{H}_{27} \mathrm{FePS}_{2} \mathrm{O} ; \% \mathrm{C}$ (calcd 62.31, found 59.81), \% $\mathrm{H}$ (calcd 5.58, found 5.58).

\subsection{Synthesis of enantiomerically pure $\left(R_{\mathrm{Fc}}\right)$ 2-thiodiphenylphosphino- (phenylsulfoxymethyl) ferrocene, $\left(R_{\mathrm{Fc}}\right)$-2a.}

The reaction was carried out using enantiomericallypure $\left(\boldsymbol{R}_{\mathbf{F c}}\right)$-1afollowing the same procedure described above for the synthesis of racemic $2 \mathbf{2 a}$. Dia1, $\left(\boldsymbol{R}_{\mathrm{Fc}}, S_{\mathbf{s}}\right)-\mathbf{2 a}:[\alpha]_{\mathrm{D}}=-100.2$; $\left(\mathrm{CHCl}_{3} . \mathrm{c}=0.49\right) ;$ Dia2, $(\boldsymbol{R} \mathrm{Fc}, \boldsymbol{R} \mathbf{s})-2 \mathbf{a}:[\alpha]_{\mathrm{D}}=+14.2 ;\left(\mathrm{CHCl}_{3} . \mathrm{c}=0.49\right)$. 


\section{6 $\left(R_{\mathrm{Fc}}\right)$ 2-thiodiphenylphosphino-(ethylsulfoxymethyl) ferrocene, $\left(\boldsymbol{R}_{\mathrm{Fc}}\right)-2 \mathrm{~b}$.}

The reaction was carried out using enantiomericallypure $\left(\boldsymbol{R}_{\mathbf{F c}}\right)$-1bfollowing the same procedure described above for the synthesis of racemic $\mathbf{2 b}$.Dia1, $\left(\boldsymbol{R}_{\mathbf{F c}}, \boldsymbol{R} \mathbf{s}\right)-\mathbf{2 b}:[\alpha]_{\mathrm{D}}=-110.2$; $\left(\mathrm{CHCl}_{3 .}\right.$ c $\left.=0.5\right) ; \operatorname{Dia2},(\boldsymbol{R} \mathbf{F c}, S \mathrm{~S})-2 \mathbf{b}:[\alpha]_{\mathrm{D}}=+32.1 ;\left(\mathrm{CHCl}_{3} . \mathrm{c}=0.505\right)$.

\section{$4.7\left(R_{\mathrm{Fc}}\right)$ 2-thiodiphenylphosphino-(tert-butylsulfoxymethyl) ferrocene, $\left(\boldsymbol{R}_{\mathrm{Fc}}\right)$-2c.}

The reaction was carried out using enantiomerically pure $\left(\boldsymbol{R}_{\mathbf{F c}}\right)$-1cfollowing the same procedure described above for the synthesis of racemic 2c.Dia1, $(\boldsymbol{R F c}, S \mathbf{s})-2 c$ : $[\alpha]_{\mathrm{D}}=-152.2$; $\left(\mathrm{CHCl}_{3} . \mathrm{c}=0.5\right) ;$ Dia2, $(\boldsymbol{R} \mathbf{F c}, \boldsymbol{R} \mathbf{s})-2 \mathrm{c}:[\alpha]_{\mathrm{D}}=+5.0 ;\left(\mathrm{CHCl}_{3} . \mathrm{c}=0.515\right)$.

\section{$4.8\left(R_{\mathrm{Fc},} S_{\mathrm{S}}\right) /\left(S_{\mathrm{Fc}}, R_{\mathrm{S}}\right)$-2-diphenylphosphino-(phenyl-sulfoxymethyl)-ferrocene,3a-Dia1.}

In a Schlenktube under argon were introduced $81 \mathrm{mg}(0.15 \mathrm{mmol})$ of racemic sulfoxide $\left(\boldsymbol{R}_{\mathbf{F c}}\right.$, $\left.S_{\mathbf{S}}\right) /\left(S_{\mathrm{Fc}}, R_{\mathbf{S}}\right)-2 \mathrm{a}-D i a 1 \quad$ in $5 \mathrm{ml}$ of toluene and $0.2 \mathrm{ml}(0.18 \mathrm{~g}, 1.1 \mathrm{mmol})$ of tris(dimethylamino)phosphine. After reflux for $3 \mathrm{~h}$, the solvent wasremoved by evaporation and the crude sulfoxide phosphine $\left(\boldsymbol{R}_{\mathrm{Fc}}, S_{\mathrm{s}}\right) /\left(\boldsymbol{S}_{\mathrm{Fc}}, \boldsymbol{R} \mathbf{s}\right)-3 \mathbf{a}-\mathrm{Dia} 1$ was obtained as an orange liquid (yield: 71mg, 93\%). ${ }^{1} \mathrm{H}$ NMR ( $\left.8,500 \mathrm{MHz} . \mathrm{CDCl}_{3}\right)$ : 7.64-7.49 (4H, m, Ph); 7.49-7.32 (6H, m, $\mathrm{Ph})$; 7.32-7.12 (5H, m, Ph); $4.43\left(1 \mathrm{H}, \mathrm{dd}(\mathrm{ABX}), \mathrm{J}_{\mathrm{HH}}=13 \mathrm{~Hz}, \mathrm{~J}_{\mathrm{HP}}=2 \mathrm{~Hz}, \underline{\mathrm{C}}_{2} \mathrm{Cp}\right) ; 4.42(1 \mathrm{H}$, broad s, substCp); $4.22\left(1 \mathrm{H}, \mathrm{t}, \mathrm{J}_{\mathrm{HH}}=2 \mathrm{~Hz}\right.$, substCp); $4.01(5 \mathrm{H}, \mathrm{s}, \mathrm{Cp}) ; 3.90(1 \mathrm{H}$, broad $\mathrm{s}$, substCp); $3.74\left(1 \mathrm{H}, \mathrm{d}(\mathrm{AB}), \mathrm{J}_{\mathrm{HH}}=13 \mathrm{~Hz}, \mathrm{C}_{2} \mathrm{Cp}\right) .{ }^{31} \mathrm{P}\left\{{ }^{1} \mathrm{H}\right\} \mathrm{NMR}\left(\delta, 202.5 \mathrm{MHz}, \mathrm{CDCl}_{3}\right)$ :22.5

\section{$4.9\left(R_{\mathrm{Fc}}, R_{\mathrm{S}}\right) /\left(S_{\mathrm{Fc}}, S_{\mathrm{S}}\right)$-2-diphenylphosphino-(phenyl-sulfoxymethyl)-ferrocene,3a-Dia2.}

Following the same procedure described abovefor the desulfurisation of $\left(\boldsymbol{R}_{\mathbf{F c}}, S_{\mathbf{S}}\right) /\left(S_{\mathbf{F c}}, R_{\mathbf{S}}\right)-\mathbf{2 a}-$ Dia1, the reaction was realized from $92 \mathrm{mg}(0.17 \mathrm{mmol})$ of racemic

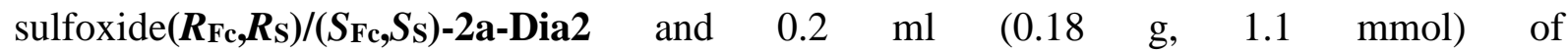
tris(dimethylamino)phosphine. The sulfoxide phosphine product $\left(\boldsymbol{R}_{\mathbf{F c}}, R_{\mathbf{s}}\right) /\left(S_{\mathbf{F c}}, S_{\mathbf{S}}\right)-3 \mathbf{a}-$ Dia2wasobtained as an orange solid (yield:73 mg,85\%). ${ }^{1} \mathrm{H}$ NMR $\left(\delta, 500 \mathrm{MHz}, \mathrm{CDCl}_{3}\right): 7.65-$ $7.5(3 \mathrm{H}, \mathrm{m}, \mathrm{Ph}) ; 7.5-7.35(6 \mathrm{H}, \mathrm{m}, \mathrm{Ph}) ; 7.35-7.2(6 \mathrm{H}, \mathrm{m}, \mathrm{Ph}) ; 4.77\left(1 \mathrm{H}, \mathrm{d}, \mathrm{J}_{\mathrm{HH}}=1 \mathrm{~Hz}\right.$, substCp); $4.43\left(1 \mathrm{H}, \mathrm{t}, \mathrm{J}_{\mathrm{HH}}=3 \mathrm{~Hz}\right.$, substCp); $4.17\left(1 \mathrm{H}, \operatorname{dd}(\mathrm{ABX}), \mathrm{J}_{\mathrm{HH}}=13 \mathrm{~Hz}, \mathrm{~J}_{\mathrm{HP}}=3 \mathrm{~Hz}\right.$, $\left.\mathrm{C}_{2} \mathrm{Cp}\right) ; 3.99(5 \mathrm{H}, \mathrm{s}, \mathrm{Cp}) ; 3.85\left(1 \mathrm{H}, \mathrm{t}, \mathrm{J}_{\mathrm{HH}}=1 \mathrm{~Hz}\right.$, substCp); $3.77\left(1 \mathrm{H}, \mathrm{d}(\mathrm{AB}), \mathrm{J}_{\mathrm{HH}}=13 \mathrm{~Hz}\right.$, $\left.\mathrm{C}_{2} \mathrm{Cp}\right) .{ }^{31} \mathrm{P}\left\{{ }^{1} \mathrm{H}\right\}$ NMR $\left(\delta, 202.5 \mathrm{MHz}, \mathrm{CDCl}_{3}\right)$ :-20.9.

\subsection{0 $\left(R_{\mathrm{Fc},}, R_{\mathrm{s}}\right) /\left(S_{\mathrm{Fc}}, S_{\mathrm{s}}\right)$-2-diphenylphosphino-(ethylsulfoxymethyl) ferrocene,3b-Dia1.}


In a Schlenktube under argon were introduced $77 \mathrm{mg}(0.157 \mathrm{mmol})$ of the racemic sulfoxide $\left(\boldsymbol{R}_{\mathbf{F c}}, \boldsymbol{R}_{\mathbf{S}}\right) /\left(\boldsymbol{S}_{\mathrm{Fc}}, \boldsymbol{S}_{\mathbf{S}}\right)-2 \mathbf{b}-\mathrm{Dia} 1 \mathrm{in} 5 \mathrm{ml}$ of toluene and $0.2 \mathrm{ml}(0.18 \mathrm{~g}, 1.1 \mathrm{mmol})$ of tris(dimethylamino)phosphine. After reflux for $2 \mathrm{~h}$, the solvent was evaporatedunder reduced pressure and the crude reaction productwas purified by column chromatography on silica usingdegased ether/dichloromethane mixture $(1 / 0$ - 1/1) as eluent. The sulfoxide phosphine product $\left(\boldsymbol{R}_{\mathbf{F c}}, \boldsymbol{R} \mathbf{S}\right) /\left(\boldsymbol{S}_{\mathbf{F c}}, S_{\mathbf{S}}\right)-3 b-D i a 1$ was obtained as an orange solid (yield:48mg, $\left.66 \%\right) .{ }^{1} \mathrm{H}$ NMR $\left(\delta, 500 \mathrm{MHz}, \mathrm{CDCl}_{3}\right): 7.62-7.50(3 \mathrm{H}, \mathrm{m}, \mathrm{Ph}) ; 7.44-7.35(2 \mathrm{H}, \mathrm{m}, \mathrm{Ph}) ; 7.28-7.08(5 \mathrm{H}, \mathrm{m}$, $\mathrm{Ph}) ; 4.62\left(1 \mathrm{H}, \mathrm{q}, \mathrm{J}_{\mathrm{HH}}=1 \mathrm{~Hz}\right.$, substCp); $4.39\left(1 \mathrm{H}, \mathrm{t}, \mathrm{J}_{\mathrm{HH}}=3 \mathrm{~Hz}\right.$, substCp); $4.15(1 \mathrm{H}, \mathrm{dd}(\mathrm{ABX})$, $\left.\mathrm{J}_{\mathrm{HH}}=14 \mathrm{~Hz}, \mathrm{~J}_{\mathrm{HP}}=2 \mathrm{~Hz}, \underline{\mathrm{C}}_{2} \mathrm{Cp}\right) ; 4.03(5 \mathrm{H}, \mathrm{s}, \mathrm{Cp}) ; 3.93\left(1 \mathrm{H}, \mathrm{d}, \mathrm{J}_{\mathrm{HH}}=1 \mathrm{~Hz}\right.$ substCp$) ; 3.76$ $\left(1 \mathrm{H}, \mathrm{d}(\mathrm{AB}), \mathrm{J}_{\mathrm{HH}}=14 \mathrm{~Hz}, \underline{\mathrm{C}}_{2} \mathrm{Cp}\right) ; 2.37\left(1 \mathrm{H}, \mathrm{dq}, \mathrm{J}_{\mathrm{HH}}=7.7 \mathrm{~Hz}, \mathrm{~J}_{\mathrm{HP}}=2.7 \mathrm{~Hz}, \underline{\mathrm{C}}_{2} \mathrm{CH}_{3}\right) ; 1.12$ $\left(1 \mathrm{H}, \mathrm{t}, \mathrm{J}_{\mathrm{HH}}=7.7 \mathrm{~Hz}, \mathrm{CH}_{2} \underline{\mathrm{H}}_{3}\right) .{ }^{31} \mathrm{P}\left\{{ }^{1} \mathrm{H}\right\} \mathrm{NMR}\left(\delta, 202.5 \mathrm{MHz}, \mathrm{CDCl}_{3}\right)$ :-22.5.MS (DCI, $\left.\mathrm{NH}_{3}\right): \mathrm{m} / \mathrm{z}=461(\mathrm{M}+1,100 \%)$.

\subsection{1 $\left(\boldsymbol{R F c}_{\mathrm{Fc}} \mathrm{S}_{\mathrm{s}}\right) /\left(\mathrm{SFc}_{\mathrm{F}}, \boldsymbol{R}_{\mathrm{s}}\right)$-2-diphenylphosphino-(ethylsulfoxymethyl) ferrocene,3b-Dia2.}

Following the same procedure described abovefor the desulfurisation of $\left(\boldsymbol{R}_{\mathrm{Fc}}, \boldsymbol{R}_{\mathrm{S}}\right) /\left(\boldsymbol{S}_{\mathrm{Fc}}, \boldsymbol{S}_{\mathbf{S}}\right)-\mathbf{2 b}$ -

Dia1, the reaction was realized from $55 \mathrm{mg}(0.112 \mathrm{mmol})$ of racemic

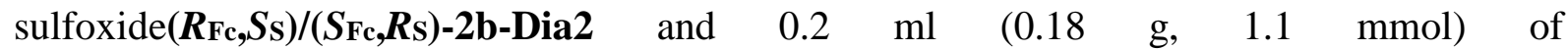
tris(dimethylamino)phosphine. The crudereaction product waspurified by column chromatography on silica usingdegased ether/methanol mixture $(1 / 0-95 / 5)$ as eluent. The sulfoxide phosphine product $\left(\boldsymbol{R}_{\mathbf{F c}}, S_{\mathbf{s}}\right) /\left(\boldsymbol{S}_{\mathbf{F c}}, \boldsymbol{R} \mathbf{s}\right)-3 b-D i a 2$ wasobtained as an orange solid (yield: $33 \mathrm{mg}, 65 \%)$. ${ }^{1} \mathrm{H}$ NMR $\left(\delta, 500 \mathrm{MHz}, \mathrm{CDCl}_{3}\right): 7.62-7.48$ (3H, m, Ph); 7.43-7.35 (3H, m, Ph); 7.30-7.13 (4H, m, Ph); $4.72\left(1 \mathrm{H}, \mathrm{d}, \mathrm{J}_{\mathrm{HH}}=1.5 \mathrm{~Hz}\right.$, substCp); $4.41\left(1 \mathrm{H}, \mathrm{t}, \mathrm{J}_{\mathrm{HH}}=1 \mathrm{~Hz}\right.$, substCp); $4.10\left(1 \mathrm{H}, \operatorname{dd}(\mathrm{ABX}), \mathrm{J}_{\mathrm{HH}}=13 \mathrm{~Hz}, \mathrm{~J}_{\mathrm{HP}}=3 \mathrm{~Hz}, \underline{\mathrm{C}}_{2} \mathrm{Cp}\right) ; 4.01(5 \mathrm{H}, \mathrm{s}, \mathrm{Cp}) ; 3.87\left(1 \mathrm{H}, \mathrm{t}, \mathrm{J}_{\mathrm{HH}}=1\right.$ $\mathrm{Hz}$, substCp); $3.72\left(1 \mathrm{H}, \mathrm{d}(\mathrm{AB}), \mathrm{J}_{\mathrm{HH}}=13 \mathrm{~Hz}, \underline{\mathrm{C}}_{2} \mathrm{Cp}\right) ; 2.45\left(1 \mathrm{H}, \mathrm{dq}, \mathrm{J}_{\mathrm{HH}}=7.6 \mathrm{~Hz}, \mathrm{~J}_{\mathrm{HP}}=3 \mathrm{~Hz}\right.$, $\left.\mathrm{C}_{2} \mathrm{CH}_{3}\right) ; 1.10\left(1 \mathrm{H}, \mathrm{t}, \mathrm{J}_{\mathrm{HH}}=7.6 \mathrm{~Hz}, \mathrm{CH}_{2} \mathrm{CH}_{3}\right) .{ }^{31} \mathrm{P}\left\{{ }^{1} \mathrm{H}\right\} \mathrm{NMR}\left(\delta, 202.8 \mathrm{MHz}, \mathrm{CDCl}_{3}\right):-21.5$.

\subsection{2 $\left(R_{\mathrm{Fc}}, S \mathrm{~s}\right) /\left(S_{\mathrm{Fc}}, R_{\mathrm{S}}\right)$-2-diphenylphosphino-(tert-butylsulfoxymethyl) ferrocene,3c-Dia1.}

In a Schlenktube under argon wereintroduced $50 \mathrm{mg}(0.096 \mathrm{mmol})$ of the sulfoxidecompound $\left(\boldsymbol{R}_{\mathrm{Fc}}, S_{\mathrm{S}}\right) /\left(\boldsymbol{S}_{\mathrm{Fc}}, \boldsymbol{R}_{\mathbf{S}}\right)-2 \mathrm{c}-D i a 1$ in $5 \mathrm{ml}$ of toluene and $0.1 \mathrm{ml}(90 \mathrm{~g}, 0.55 \mathrm{mmol})$ of tris(dimethylamino)phosphine. After reflux for $2 \mathrm{~h}$, the solvent wasremoved and the crudereaction product waspurified by column chromatography on silica usingdegased ether/dichloromethane mixture $(1 / 1)$ as eluent. the sulfoxide phosphine product $\left(\boldsymbol{R}_{\mathbf{F c}}, S_{\mathbf{S}}\right) /\left(\boldsymbol{S}_{\mathbf{F c}}, \boldsymbol{R} \mathbf{s}\right)-3 c-D i a 1$ wasobtained as an orange liquid (yield: $\left.36 \mathrm{mg}, 76 \%\right) .{ }^{1} \mathrm{H}$ NMR $\left(\delta, 500 \mathrm{MHz}, \mathrm{CDCl}_{3}\right)$ :7.58-7.42 (2H, m, Ph); 7.43-7.32 (3H, m, Ph); 7.30-7.18 (3H, m, Ph); 
7.18-7.05 (2H, m, Ph); 4.79 (1H, s broad, substCp); 4.34 (1H, t, $\mathrm{J}_{\mathrm{HH}}=1 \mathrm{~Hz}$, substCp); 4.08 $(5 \mathrm{H}, \mathrm{s}, \mathrm{Cp}) ; 3.82\left(1 \mathrm{H}, \mathrm{t}, \mathrm{J}_{\mathrm{HH}}=1 \mathrm{~Hz}, \operatorname{substCp}\right) ; 3.73\left(1 \mathrm{H}, \mathrm{dd}(\mathrm{ABX}), \mathrm{J}_{\mathrm{HH}}=12 \mathrm{~Hz}, \mathrm{~J}_{\mathrm{HP}}=2 \mathrm{~Hz}\right.$, $\left.\mathrm{C}_{2} \mathrm{Cp}\right) ; 3.30\left(1 \mathrm{H}, \mathrm{d}(\mathrm{AB}), \mathrm{J}_{\mathrm{HH}}=12 \mathrm{~Hz}, \mathrm{C}_{2} \mathrm{Cp}\right) ; 1.2\left(9 \mathrm{H}, \mathrm{s}, \mathrm{C}\left(\mathrm{C}_{3}\right)_{3}\right) .{ }^{31} \mathrm{P}\left\{{ }^{1} \mathrm{H}\right\} \mathrm{NMR}$ $\left(\delta, 202.8 \mathrm{MHz}, \mathrm{CDCl}_{3}\right):-20.6$.

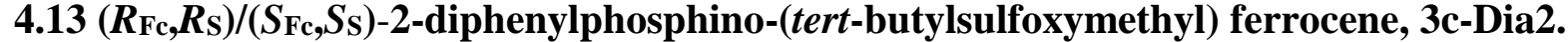

Following the same procedure described abovefor the desulfurisation of $\left(\boldsymbol{R}_{\mathbf{F c}}, S_{\mathbf{S}}\right) /\left(S_{\mathbf{F c}}, R_{\mathbf{S}}\right)-\mathbf{2 c}-$ Dia1, the reaction was realized from $70 \mathrm{mg}(0.135 \mathrm{mmol})$ of the sulfoxide $\operatorname{compound}(\boldsymbol{R} \mathbf{F c}, \boldsymbol{R} \mathbf{s}) /\left(S_{\mathbf{F c}, S \mathbf{s})-2 c-D i a 2}\right.$ and $\quad 0.2 \quad \mathrm{ml} \quad(0.18 \quad \mathrm{~g}, \quad 1.1 \quad \mathrm{mmol}) \quad$ of tris(dimethylamino)phosphine. The sulfoxide phosphine product $\left(\boldsymbol{R}_{\mathbf{F c}}, \boldsymbol{R}_{\mathbf{S}}\right) /\left(\boldsymbol{S}_{\mathbf{F c}}, S_{\mathbf{S}}\right)-3 c-D i a 2$ wasobtained as an orange solid (yield: $57 \mathrm{mg}, 86 \%) .{ }^{1} \mathrm{H}$ NMR $\left(\delta, 500 \mathrm{MHz}, \mathrm{CDCl}_{3}\right): 7.57-7.46$ $(3 \mathrm{H}, \mathrm{m}, \mathrm{Ph}) ; 7.42-7.34$ (2H, m, Ph); 7.28-7.12 (5H, m, Ph); $4.78\left(1 \mathrm{H}, \mathrm{d}, \mathrm{J}_{\mathrm{HH}}=2 \mathrm{~Hz}\right.$, substCp); $4.38\left(1 \mathrm{H}, \mathrm{t}, \mathrm{J}_{\mathrm{HH}}=1 \mathrm{~Hz}\right.$, substCp$) ; 4.06\left(1 \mathrm{H}, \mathrm{dd}(\mathrm{ABX}), \mathrm{J}_{\mathrm{HH}}=13 \mathrm{~Hz}, \mathrm{~J}_{\mathrm{HP}}=2 \mathrm{~Hz}, \mathrm{CH}_{2} \mathrm{Cp}\right) ; 4.04$ $(5 \mathrm{H}, \mathrm{s}, \mathrm{Cp}) ; 3.75\left(1 \mathrm{H}, \mathrm{d}, \mathrm{J}_{\mathrm{HH}}=1 \mathrm{~Hz}\right.$, substCp); $3.36\left(1 \mathrm{H}, \mathrm{d}(\mathrm{AB}), \mathrm{J}_{\mathrm{HH}}=13 \mathrm{~Hz}, \mathrm{C}_{2} \mathrm{Cp}\right) ; 1.05$ $\left(9 \mathrm{H}, \mathrm{s}, \mathrm{C}\left(\mathrm{C}_{3}\right)_{3}\right) .{ }^{31} \mathrm{P}\left\{{ }^{1} \mathrm{H}\right\}$ NMR $\left(\delta, 202.8 \mathrm{MHz}, \mathrm{CDCl}_{3}\right):-20.9$.

\section{X-ray analyses}

A single crystal of each compound was mounted under inert perfluoropolyether at the tip of a glass fibre and cooled in the cryostream of either an Oxford-Diffraction XCALIBUR CCD diffractometer for 2a-Dia1, 2a-Dia2, 2b-Dia1 and3a-Dia2ora Stoe IPDS diffractometer for 2c-Dia1, 2c-Dia2 and 4a. Data were collected using the monochromatic MoK $\alpha$ radiation $(\lambda=0.71073)$.

The structures were solved by direct methods $\left(\operatorname{SIR} 97^{21}\right)$ and refined by least-squares procedures on $F^{2}$ using SHELXL-97. ${ }^{22}$ All $\mathrm{H}$ atoms attached to carbon were introduced in calculation in idealised positions and treated as riding models. The drawing of the molecules was realised with the help of ORTEP32. ${ }^{24}$ Crystal data and refinement parameters are shown in Table 2. 
Table 2.Crystal data and structure refinement parameters.

\begin{tabular}{|c|c|c|c|}
\hline Identification code & 2a-Dia1 & 2a-Dia2 & 2b-Dia1 \\
\hline Empirical formula & $\mathrm{C}_{29} \mathrm{H}_{25} \mathrm{FeOPS}{ }_{2}$ & $\mathrm{C}_{29} \mathrm{H}_{25} \mathrm{FeOPS}{ }_{2}$ & $\mathrm{C}_{25} \mathrm{H}_{25} \mathrm{FeOPS}_{2}$ \\
\hline Formula weight & 540.43 & 540.43 & 492.39 \\
\hline Temperature, $\mathrm{K}$ & $180(2)$ & $180(2)$ & $180(2)$ \\
\hline Wavelength, $\AA$ & 0.71073 & 0.71073 & 0.71073 \\
\hline Crystal system & Monoclinic & Monoclinic & Orthorhombic \\
\hline Space group & $\mathrm{Cc}$ & $\mathrm{P} 22_{1} / \mathrm{a}$ & Pcab \\
\hline $\mathrm{a}, \AA$ & $14.5928(10)$ & $12.812(2)$ & $8.1197(6)$ \\
\hline $\mathrm{b}, \AA$ & $18.4079(11)$ & $9.4172(16)$ & $16.9489(11)$ \\
\hline $\mathrm{c}, \AA$ & $9.4244(6)$ & $21.896(4)$ & $33.823(2)$ \\
\hline$\alpha,{ }^{\circ}$ & 90.0 & 90.0 & 90.0 \\
\hline$\beta,{ }^{\circ}$ & $93.858(5)$ & $104.832(16)$ & 90.0 \\
\hline$\gamma,^{\circ}$ & 90.0 & 90.0 & 90.0 \\
\hline Volume, $\AA^{3}$ & $2525.9(3)$ & $2553.8(8)$ & $4654.7(5)$ \\
\hline $\mathrm{Z}$ & 4 & 4 & 8 \\
\hline Density (calcd), $\mathrm{Mg} / \mathrm{m}^{3}$ & 1.421 & 1.322 & 1.405 \\
\hline Absorption coefficient, $\mathrm{mm}^{-1}$ & 0.847 & 0.754 & 0.911 \\
\hline $\mathrm{F}(000)$ & 1120 & 1056 & 2048 \\
\hline Crystal size, $\mathrm{mm}^{3}$ & $0.45 \times 0.42 \times 0.2$ & $0.16 \times 0.10 \times 0.05$ & $0.52 \times 0.39 \times 0.12$ \\
\hline Theta range, ${ }^{\circ}$ & 3.56 to 27.81 & 2.71 to 26.03 & 3.32 to 26.37 \\
\hline Reflections collected & 9974 & 18130 & 33297 \\
\hline Independent reflections [R(int)] & $4487(0.0242)$ & $5055(0.1527)$ & $4751(0.0634)$ \\
\hline Completeness, $\%$ & 99.7 & 99.7 & 99.8 \\
\hline Absorption correction & Multiscan & Multi-scan & Multi-scan \\
\hline Max. , min. transmission & 0.8415 and 0.6881 & 1.0500 and 0.8078 & 0.8230 and 0.6750 \\
\hline Refinement method & $\mathrm{F}^{2}$ & $\mathrm{~F}^{2}$ & $\mathrm{~F}^{2}$ \\
\hline Data / restraints / parameters & $4487 / 2 / 308$ & $5055 / 0 / 307$ & $4751 / 0 / 272$ \\
\hline Goodness-of-fit on $\mathrm{F}^{2}$ & 1.055 & 0.892 & 1.081 \\
\hline Final R indices [I>2sigma(I)] & $0.0278,0.0642$ & $0.0662,0.1564$ & $0.0514,0.1035$ \\
\hline $\mathrm{R}$ indices (all data) & $0.0305,0.0656$ & $0.1713,0.2309$ & $0.0704,0.1118$ \\
\hline Absolute structure parameter & $0.019(14)$ & & \\
\hline Largest diff. peak and hole, e. $\AA^{-3}$ & 0.326 and -0.219 & 0.711 and -0.865 & 0.417 and -0.367 \\
\hline
\end{tabular}


Table 1. Crystal data and structure refinement(Contd.).

\begin{tabular}{|c|c|c|c|}
\hline Identification code & 2c-Dia1 & 2c-Dia2 & 3a-Dia2 \\
\hline Empirical formula & $\mathrm{C}_{27} \mathrm{H}_{29} \mathrm{FeOPS}_{2}$ & $\mathrm{C}_{27} \mathrm{H}_{29} \mathrm{FeOPS}{ }_{2}$ & $\mathrm{C}_{29} \mathrm{H}_{25} \mathrm{FeOPS}$ \\
\hline Formula weight & 520.44 & 520.44 & 508.37 \\
\hline Temperature, $\mathrm{K}$ & $180(2)$ & $180(2)$ & $180(2)$ \\
\hline Wavelength, $\AA$ & 0.71073 & 0.71073 & 0.70930 \\
\hline Crystal system & Monoclinic & Monoclinic & Triclinic \\
\hline Space group & $\mathrm{Cc}$ & $\mathrm{P} 2_{1}$ & $\mathrm{P}-1$ \\
\hline $\mathrm{a}, \AA$ & $11.8130(12)$ & $14.7673(14)$ & $7.8035(8)$ \\
\hline $\mathrm{b}, \AA$ & $14.8503(12)$ & $10.1912(7)$ & $9.3075(9)$ \\
\hline $\mathrm{c}, \AA$ & $13.9653(16)$ & $17.0533(18)$ & $17.5040(15)$ \\
\hline$\alpha,{ }^{\circ}$ & 90.0 & 90.0 & $79.331(7)$ \\
\hline$\beta,^{\circ}$ & $91.394(13)$ & $98.030(12)$ & $87.727(7)$ \\
\hline$\gamma,{ }^{\circ}$ & 90.0 & 90.0 & $77.299(8)$ \\
\hline Volume, $\AA^{3}$ & $2449.2(4)$ & $2541.3(4)$ & $1218.8(2)$ \\
\hline $\mathrm{Z}$ & 4 & 4 & 2 \\
\hline Density (calcd), $\mathrm{Mg} / \mathrm{m}^{3}$ & 1.411 & 1.360 & 1.385 \\
\hline Absorption coefficient, $\mathrm{mm}^{-1}$ & 0.870 & 0.838 & 0.790 \\
\hline $\mathrm{F}(000)$ & 1088 & 1088 & 528 \\
\hline Crystal size, $\mathrm{mm}^{3}$ & $0.76 \times 0.22 \times 0.2$ & $0.56 \times 0.52 \times 0.02$ & $0.35 \times 0.32 \times 0.2$ \\
\hline Theta range, ${ }^{\circ}$ & 2.62 to 26.04 & 2.33 to 26.08 & 3.47 to 28.22 \\
\hline Reflections collected & 11892 & 21668 & 10784 \\
\hline Independent reflections [R(int)] & $4769(0.0442)$ & $9539(0.0730)$ & $6009(0.0237)$ \\
\hline Completeness, $\%$ & 98.8 & 97.7 & 99.1 \\
\hline Absorption correction & Multi-scan & Multi-scan & Multi-scan \\
\hline Max. , min. transmission & 0.943 and 0.610 & 0.8058 and 0.7391 & 0.7521 and 0.6862 \\
\hline Refinement method & $\mathrm{F}^{2}$ & $\mathrm{~F}^{2}$ & $\mathrm{~F}^{2}$ \\
\hline Data / restraints / parameters & 4769 / 2 / 292 & $9539 / 1 / 584$ & $6009 / 0 / 298$ \\
\hline Goodness-of-fit on $\mathrm{F}^{2}$ & 1.025 & 0.843 & 0.991 \\
\hline Final R indices $[\mathrm{I}>2 \operatorname{sigma}(\mathrm{I})]$ & $0.0302,0.0778$ & $0.0379,0.0611$ & $0.0389,0.1012$ \\
\hline $\mathrm{R}$ indices (all data) & $0.0314,0.0785$ & $0.0716,0.0685$ & $0.0507,0.1067$ \\
\hline Absolute structure parameter & $-0.004(11)$ & $0.284(14)$ & \\
\hline Largest diff. peak and hole, e. $\AA^{-3}$ & 0.318 and -0.384 & 0.328 and -0.425 & 1.418 and -0.385 \\
\hline
\end{tabular}


Table 1.Crystal data and structure refinement(Contd.).

\begin{tabular}{|c|c|}
\hline Identification code & $4 \mathbf{a}$ \\
\hline Empirical formula & $\mathrm{C}_{29} \mathrm{H}_{25} \mathrm{Cl}_{2} \mathrm{FeOPPt} \mathrm{S}$ \\
\hline Formula weight & 774.36 \\
\hline Temperature, $\mathrm{K}$ & $180(2)$ \\
\hline Wavelength, $\AA$ & 0.71073 \\
\hline Crystal system & Monoclinic \\
\hline Space group & $\mathrm{P} 2_{1} / \mathrm{n}$ \\
\hline $\mathrm{a}, \AA$ & $18.6057(14)$ \\
\hline $\mathrm{b}, \AA$ & $7.5780(7)$ \\
\hline $\mathrm{c}, \AA$ & $19.3027(14)$ \\
\hline$\alpha,{ }^{\circ}$ & 90.0 \\
\hline$\beta,^{\circ}$ & $107.659(8)$ \\
\hline$\gamma,^{\circ}$ & 90.0 \\
\hline Volume, $\AA^{3}$ & 2593.3(4) \\
\hline $\mathrm{Z}$ & 4 \\
\hline Density (calcd), $\mathrm{Mg} / \mathrm{m}^{3}$ & 1.983 \\
\hline Absorption coefficient, $\mathrm{mm}^{-1}$ & 6.319 \\
\hline $\mathrm{F}(000)$ & 1504 \\
\hline Crystal size, $\mathrm{mm}^{3}$ & $0.18 \times 0.08 \times 0.08$ \\
\hline 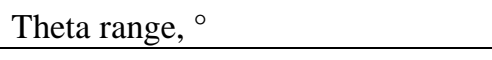 & 2.21 to 26.06 \\
\hline Reflections collected & 24800 \\
\hline Independent reflections [R(int)] & $5081(0.0562)$ \\
\hline Completeness, $\%$ & 99.0 \\
\hline Absorption correction & Multi-scan \\
\hline Max., min. transmission & 0.5281 and 0.4506 \\
\hline Refinement method & $\mathrm{F}^{2}$ \\
\hline Data / restraints / parameters & $5081 / 0 / 325$ \\
\hline Goodness-of-fit on $\mathrm{F}^{2}$ & 0.981 \\
\hline Final $\mathrm{R}$ indices $[\mathrm{I}>2 \operatorname{sigma}(\mathrm{I})]$ & $0.0289,0.0585$ \\
\hline $\mathrm{R}$ indices (all data) & $0.0445,0.0624$ \\
\hline \multicolumn{2}{|l|}{ Absolute structure parameter } \\
\hline Largest diff. peak and hole, e. $\AA^{-3}$ & 0.907 and -0.927 \\
\hline
\end{tabular}


${ }^{1}$ Imamoto,T.; Sugita,K.; Yoshida, K. J. Am. Chem. Soc. 2005, 127,11934.
${ }^{2}$ Knowles, W. S.; Sabacky, M. J.; Vineyard,B. D.; Weinkauff, D. J. J.Am. Chem. Soc. 1975, 97,2567.

${ }^{3}$ For recent reviews, see: (a) Masdeu-Bulto, A. M.; Dieguez, M.; Martin, E.; Gomez, M. Coord. Chem. Rev. 2003, 159; (b) Pellissier, H. Tetrahedron2007, 63, 1297; (c) Mellah, M.; Voituriez, A.; Schulz, E. Chem. Rev. 2007, 107, 5133; (d) Malacea, R.; Manoury, E. in "Phosphorus-Sulfur Ligands» from "Phosphorus Ligands in Asymmetric Catalysis" edited byArminBörner (Wiley-VCH, Weinheim, Allemagne) 2005, 749; (e) Lam, F. L.; Kwong, F. Y.; Chan, A. S. C. Chem. Commun.2010, 46, 4649.

${ }^{4}$ Selected references (a) Albinati, A.; Pregosin, P. S.; Wick,K. Organometallics1996, 15, 2419; (b) Spencer, J.;Gramlich, V.; Hausel, R.; Togni, R. Tetrahedron: Asymmetry1996, 7, 41; (c) Enders, D.;Peters, R.; Lochtman, R.; Raabe, G.; Runsink, J.; Bats, J.W. Eur. J. Org. Chem. 2000, 3399; (d) Verdaguer, X.;Moyano, A.; Pericas, M. A.; Riera, A.; Maestro, M. A.;Mahia, J. J. Am. Chem. Soc. 2000, 122, 10242; (e) Evans, D. A.; Michael, F. E.; Tedrow, J. S.; Campos, K.R. J. Am. Chem. Soc. 2003, 125, 3534; (f) Nakano,H.; Yokohama, J.; Okuyama, Y.; Fujita, R.; Hongo, H.Tetrahedron: Asymmetry2003, 14, 2361; (g) Tu, T.;Zhou, Y.-G.; Hou, X.-L.; Dai, L.X.; Dong, X.-C.; Yu,Y.-H.; Sun, J. Organometallics2003, 22, 1255; (h) Priego, J.; Mancheño, O. G.; Cabrera, S.; Arrayás, R.; Llamas, T.; Carretero, J. C. Chem. Commun. 2002, 2512; (k) Mancheno, O. G.; Priego, J.; Cabrera, S.; Arrayas, R. G.;Llamas, T.; Carretero, J. C. J. Org. Chem. 2003, 68, 3679. (1) Zeng, W.; Zhou,Y.G. Tet. Lett.2007, 48, 4619.

${ }^{5}$ (a) Alcock, N.X.; Brown, J.M.; Evans, P.L. J. Organomet. Chem. 1988, 356, 233; (b) Quek, G.H.; Leung, P.H.; Mok, K.F. Inorg.Chim.Acta1995, 239, 185

${ }^{6}$ (a) Sola, J. ; Revs, M. ; Riera, A.; Verdaguer, X. Angew. Chem. Int. Ed. 2007, 46, 5020 ; b) Reves, M.; Achard, T.; Sola, J.; Riera,A.; Verdaguer, X. J. Org. Chem. 2008, 73, 7080; c) Ji, Y.; Riera, A.; Verdaguer, X.Org. Lett.2009,11,19 4346.

${ }^{7}$ (a) Chooi, S.Y.M.; Leung , P.H.; Mok, K.F. Inorg.Chim.Acta1993, 205, 245-247; (b) Hiroi, K.; Suzuki, Y.; Kawagishi, R. Tet.Lett.1999, 40, 715; (c) Xing, J., Cao, P., Liao, J. Tetrahedron: Asymmetry2012, 23, 527-535.

${ }^{8}$ Ferrer, C.; Riera, A.; Verdaguer, X. Organometallics 2009, 28, 4571.

${ }^{9}$ Solà,J.; Riera, A. ; Verdaguer, X. J. Organomet. Chem. 2010,695, 2377.

10 (a) Riant, O.;Argouarch, G.;Guillaneux, D.; Samuel, O.;Kagan, H.B.J. Org. Chem. 1998, 63, 3511; (b) Cheemala, M.N ; Knochel, P. Org. Lett.2007, 9, 3089; (c) Zhang, H.L.;Hou, X.L.; Dai, L.X.;Luo, Z.B.Tetrahedron: Asymmetry2007, 18, 224.

${ }^{11}$ (a) Routaboul, L.; Vincendeau, S.; Daran, J.C.; Manoury, E. Tetrahedron: Asymmetry2005, 16, 2685; (b) Diab, L., Gouygou, M., Manoury, E., Kalck P., Urrutigoïty, M. Tet. Lett. 2008, 49, 5186. (c) Le Roux, E.; Malacea, R.; Manoury, E.; Poli, R.; Gonsalvi, L.;Peruzzini, M. Adv. Synth Catal.2007, 349, 309; (d) Manoury, E., Poli, R. "Phosphine-Containing Planar Chiral ferrocenes: Synthesis, Coordination Chemistry and Applications to Asymmetric Catalysis."n the Series: Catalysis by Metal Complexes (CMCO), Volume 36 (Phosphorus Chemistry: Catalysis and Material Science Applications), edited by Maurizio Peruzzini and Luca Gonsalvi (Springer Verlag, Germany) 2011, p121.

${ }^{12}$ Mateus, N. ;Routaboul, L. ;Daran, J.-C. ; Manoury, E. J. Organomet. Chem. 2006, 691, 2297.

${ }^{13} \mathrm{Xu}$, W.L.; Zheng Li, Y.; Shan Zhang, Q.; Sun Zhu, H.Synthesis2004, 2, 227. ${ }^{14}$ Weigand, W.; Bosl, G.ZeitschriftfuerNaturforschung, Chemical Sciences1992, 47, 1165.

${ }^{15}$ Di Furia, F.; Modena, G.;Seraglia, R.Synthesis, 1984, 325.

${ }^{16}$ Conte, V.; Di Furia, F. ;Lucini, G. ; Modena,G. Tet. Lett.,1989, 30, 4859.

17 (a) Calligaris, M.Coord. Chem. Rev. 2004, 248, 351; (b) Kagan, H. B.; Ronan, B., Rev. Heteroatom Chem. Rev.1992, 248, 92.

${ }^{18}$ Anderson, G. K.; Kumar,R. Inorg.Chem.1984, 23, 4064.

${ }^{19}$ Cremer, D.;Pople, J.A.J.Am.Chem.Soc.1975, 97, 1354.

${ }^{20}$ Malacea, R.; Manoury, E.; Daran, J.C.; Poli, R.J. Molec.Str.2008, 890, 249.

${ }^{21}$ (a) Malacea, R.; Manoury, E.; Routaboul, L.; Daran, J.C.; Poli, R.; Dunne, J.P. ; Withood, A.C.; Godard,C.; Duckett, S.B. Eur. J. Inorg. Chem. 2006, 9, 1803; (b) Malacea, R., Daran, J.-C., Duckett, S. B., Dunne, J. P., Manoury, E., Poli, R., Withwood, A. C. Dalton Trans.2006, 3350.

${ }^{18}$ Altomare, A.; Burla, M.C.; Camalli, M.; Cascarano, G.L.; Giacovazzo, C.; Guagliardi, A.; Moliterni, A.G.G. ; Polidori, G.; Spagna R. J. Appl. Cryst.1999, 32, 115.

${ }^{19}$ Sheldrick, G.M. ActaCryst. 2008, A64, 112.

${ }^{20}$ Farrugia, L.J. J. Appl. Cryst.1999, 32, 837.

${ }^{21}$ Farrugia, L.J. J. Appl. Cryst.1997, 30, 565.

${ }^{22}$ Spek, A.L.J. Appl. Cryst.2003, 36, 7. 